TRANSACTIONS OF THE

AMERICAN MATHEMATICAL SOCIETY

Volume 364, Number 9, September 2012, Pages 4987-5014

S 0002-9947(2012)05592-9

Article electronically published on April 6, 2012

\title{
AN EXTREMELY NON-HOMOGENEOUS WEAK HILBERT SPACE
}

\author{
SPIROS A. ARGYROS, KEVIN BEANLAND, AND THEOCHARIS RAIKOFTSALIS
}

\begin{abstract}
We construct a weak Hilbert Banach space such that for every block subspace $Y$ every bounded linear operator on $Y$ is of the form $D+S$, where $S$ is a strictly singular operator and $D$ is a diagonal operator. We show that this yields a weak Hilbert space whose block subspaces are not isomorphic to any of their proper subspaces.
\end{abstract}

\section{INTRODUCTION}

The weak Hilbert spaces form a class of Banach spaces including Hilbert spaces that share many of their important properties. We recall their definition. An infinite dimensional Banach space $X$ is called a weak Hilbert space if there exist positive numbers $\delta, C$ such that every finite dimensional space $E \subset X$ contains a subspace $F \subset E$ such that $\operatorname{dim} F \geq \delta \operatorname{dim} E$, the Banach-Mazur distance between $F$ and $\ell_{2}^{\operatorname{dim} F}$ is at most equal to $C$ and there is a projection $P: X \rightarrow F$ with $\|P\| \leq C\left(\ell_{2}^{n}\right.$ denotes the Hilbert space of dimension $\left.n\right)$. The above definition finds its origins in the seminal works of V. Milman and G. Pisier [25] and G. Pisier 27. However, the book of G. Pisier [28] remains the most comprehensive reference for weak Hilbert spaces. In [28, one can find numerous characterizations and an in-depth discussion of the properties of these spaces. All subspaces, quotients and duals of weak Hilbert spaces are themselves weak Hilbert. The Fredholm theory as developed by Grothendieck [18] works in weak Hilbert spaces as well as Hilbert spaces, and W.B. Johnson (unpublished; see [28]) showed that all weak Hilbert spaces are superreflexive.

When one considers the rich structure and geometry of a Hilbert space, it is natural to ask what kind of geometry a weak Hilbert space must possess. In particular, it is very interesting to investigate how divergent the global geometry of such a space can be when compared with the local Hilbertian structure. The most significant step in this direction was made by W.B. Johnson in 19, where it was shown that the 2-convexification of the modified Tsirelson space is a weak Hilbert space with no subspace isomorphic to a Hilbert space. Our aim in the present paper is to construct a weak Hilbert space having a quite divergent structure from that of a Hilbert space. Namely, we construct a Banach space $\mathfrak{X}_{w h}$ with an unconditional basis $\left(e_{n}\right)_{n}$ that has the following, not necessarily independent, properties:

(1) $\mathfrak{X}_{w h}$ is a weak Hilbert space.

(2) The space $\mathfrak{X}_{w h}$ is asymptotic $\ell_{2}$ for vectors with disjoint support or strongly asymptotic $\ell_{2}$.

Received by the editors February 7, 2011.

2010 Mathematics Subject Classification. Primary 46B20, 46B06.

Key words and phrases. Weak Hilbert spaces.

(c) 2012 American Mathematical Society Reverts to public domain 28 years from publication 4987 
(3) For every block subspace $Y$ of $\mathfrak{X}_{w h}$ every operator in $\mathcal{L}(Y)$ takes the form $\left.D\right|_{Y}+S$, where $D e_{n}=\lambda_{n} e_{n}$ for some scalar sequence $\left(\lambda_{n}\right)$ and $S \in \mathcal{L}(Y)$ is strictly singular.

(4) Every block subspace $Y$ of $\mathfrak{X}_{w h}$ is not isomorphic to any of its proper subspaces.

(5) The space $\mathfrak{X}_{w h}$ does not contain a quasi-minimal subspace.

(6) No disjointly supported subspaces of $\mathfrak{X}_{w h}$ are isomorphic. Using the terminology from [14] the space $\mathfrak{X}_{w h}$ is tight by support.

In the above and herein, we use $\mathcal{L}(Y)$ to denote the Banach space of bounded linear operators on $Y$. An operator $S \in \mathcal{L}(Y)$ is strictly singular if its restriction to any infinite dimensional subspace is not an isomorphism. In the sequel, we call an operator $D$ diagonal if $D e_{n}=\lambda_{n} e_{n}$ for some scalar sequence $\left(\lambda_{n}\right)$ and some a priori fixed basis $\left(e_{n}\right)$. Although the space we construct is built over the field of real numbers, by applying exactly the same methods one can naturally extend the construction to that of space with the same properties defined over the field of complex numbers. In both cases, we correlate the spectrum of an operator $T \in \mathcal{L}(Y)$ with that of the diagonal operator $D$, where $T=D+S$. We also recall that a space $X$ is called quasi-minimal if it does not contain a pair of totally incomparable subspaces.

There are several criteria for showing that a space is weak Hilbert. One of them concerns spaces with a Schauder basis and relates to how the norm behaves on disjointly supported vectors. More precisely, N. J. Nielsen and N. TomczakJaegermann in [26], by applying theorems of W.B. Johnson [20], show that a space with a basis is weak Hilbert if the basis is asymptotic $\ell_{2}$ for vectors with disjoint support. A good reference for this proof is 2]. We recall the definition of this notion here. A space $X$ with a basis $\left(e_{i}\right)$ is an asymptotic $\ell_{2}$ for vectors with disjoint supports if there is a $C \geq 1$ such that for every $n \in \mathbb{N}$ and every sequence of disjointly supported vectors $\left(x_{i}\right)_{i=1}^{n}$ with $n \leq \operatorname{supp} x_{i}$ for $i \leq n,\left(x_{i}\right)_{i=1}^{n}$ is $C$-equivalent to the unit vector basis of $\ell_{2}$. This property is also called strongly asymptotic $\ell_{2}$. The notion of strongly asymptotic $\ell_{p}$ spaces was introduced by Tcaciuc in [30] and extensively studied in the paper [12. Using this condition, Edgington [13], and then later Androulakis, Casazza, and Kutzarova [2, constructed non-trivial weak Hilbert spaces each with an unconditional basis and saturated with copies of $\ell_{2}$. The definition of the space $\mathfrak{X}_{w h}$ presented in this paper utilizes a type of modified mixed Tsirelson saturation method which yields the aforementioned property. In the next section we give a description of the norm of $\mathfrak{X}_{w h}$ and further discuss some of its critical properties. We note that in [14 they construct a space that is strongly asymptotic $\ell_{1}$ and has a property they call tight by support. The space $\mathfrak{X}_{w h}$ is the first example of a strongly asymptotic $\ell_{2}$ space that is tight by support. Note that the properties of the operators on $\mathfrak{X}_{w h}$ easily imply that $\mathfrak{X}_{w h}$ does not admit a complex structure. This solves a problem in [3].

An announcement of the results of this paper have appeared in [4].

\section{Description of $\mathfrak{X}_{w h}$}

Let $c_{00}$ denote the vector space of the finitely supported scalar sequences and $\left(e_{n}\right)$ denote the unit vector basis of $c_{00}$. For $x=\sum_{i=1}^{\infty} a_{i} e_{i} \in c_{00}$, let $\operatorname{supp} x=\{i \in$ $\left.\mathbb{N}: a_{i} \neq 0\right\}$ and $\operatorname{ran} x$ be the smallest interval containing $\operatorname{supp} x$. For $E, F \subset \mathbb{N}$, write $E<F$ if $\max E<\min F$ or either $E$ or $F$ is empty. For $x, y \in c_{00}$ we write 
$x<y$ and call $x, y$ successive if $\operatorname{ran} x<\operatorname{ran} y$. We write $n<x$ if $\{n\}<x$. For $E \subset \mathbb{N}$ and $\sum_{i=1}^{\infty} a_{i} x_{i} \in c_{00}$ let $E x=\sum_{i \in E} a_{i} x_{i}$.

The definition of the space $\mathfrak{X}_{w h}$ uses an injective function with range a subset of the natural numbers or what has been referred to as a coding function. Coding functions were first introduced by B. Maurey and H.P. Rosenthal in 24, where they construct a weakly null sequence with no unconditional subsequence. They have become a ubiquitous component of constructions of spaces with few operators and, as in our case, few symmetries. In [16, W.T. Gowers constructs the first example of a space $\mathfrak{X}_{g u}$ not isomorphic to any of its hyperplanes. The operators on this space take the form $D+S$, where $D$ is diagonal and $S$ is strictly singular (cf. [17). This is the first example of a space whose construction uses a coding function but which had an unconditional basis; our construction is similar in this way. On the other hand, an important new feature of our construction is that it admits an implicit description that is similar to the modified mixed Tsirelson spaces described in [2, 5, 6, 13.

To help the reader better understand what is meant by this, we state the implicit equations that the norm satisfies. For $n \in \mathbb{N}$, let $\mathcal{S}_{n}^{M}$ denote the modified Schreier family of order $n$ (see Section 3 for definitions). A finite family $\left(E_{i}\right)_{i=1}^{d}$ of pairwise disjoint finite subsets of $\mathbb{N}$ is called $\mathcal{S}_{n}^{M}$ allowable if $\left\{\min E_{i}\right\}_{i=1}^{d} \in \mathcal{S}_{n}^{M}$. The norm requires two increasing sequences $\left(m_{j}\right)_{j=0}^{\infty}$ and $\left(n_{i}\right)_{i=0}^{\infty}$ satisfying certain growth conditions, and so let us fix these throughout. The norm of $\mathfrak{X}_{w h}$ is the completion of $c_{00}$ in the norm $\|x\|=\max \left\{\sup \left\{\|x\|_{j}: j \in \mathbb{N} \cup\{0\}\right\},\|x\|_{\infty}\right\}$, where the norms $\|\cdot\|_{j}$ satisfy the following implicit formulas:

$\|x\|_{2 j}=\sup \left\{\frac{1}{m_{2 j}}\left(\sum_{i=1}^{k}\left\|E_{i} x\right\|^{2}\right)^{\frac{1}{2}}:\left(E_{i}\right)_{i=1}^{k}\right.$ is $S_{n_{2 j}}^{M}$ allowable $\}$,

$\|x\|_{2 j+1}=\sup \left\{\frac{1}{m_{2 j+1}}\left(\sum_{i=1}^{k}\left\|E_{i} x\right\|_{2 j_{i}}^{2}\right)^{\frac{1}{2}}:\left(E_{i}, 2 j_{i}\right)_{i=1}^{k}\right.$ is an $S_{n_{2 j+1}}^{M}-\sigma$ special sequence $\}$.

The $\|\cdot\|_{2 j+1}$ norms and special sequences are the key ingredients in showing that our space has the desired asymmetry. The special sequences impose the nonhomogeneous structure on the space. We briefly outline some properties of special sequences; the exact definition can be found in Section 3. Readers familiar with previous constructions will notice many similarities (such as the 'tree-like property'). Let $N_{1}$ and $N_{2}$ be infinite subsets of $\mathbb{N}$ with $\mathbb{N}=N_{1} \cup N_{2}$. Let

$$
\begin{aligned}
\Sigma=\left\{\left(E_{i}, 2 j_{i}\right)_{i=1}^{n}:\right. & E_{i} \cap E_{j}=\emptyset \text { and } j_{1}<j_{2}<\cdots<j_{n} \\
& \text { with } \left.j_{1} \in N_{1} \text { and } j_{i} \in N_{2} \text { for } i>1\right\} .
\end{aligned}
$$

Special sequences $\left(E_{i}, 2 j_{i}\right)_{i=1}^{n} \in \Sigma$ have two important properties:

(i) (Extension property) Suppose $\left(E_{i}, 2 j_{i}\right)_{i=1}^{n}$ is a special sequence and $E \subset$ $\mathbb{N}$ finite with $E \cap\left(\bigcup_{i=1}^{n} E_{i}\right)=\emptyset$. Then there exists a $j \in \mathbb{N}$ such that $\left(\left(E_{i}, 2 j_{1}\right), \ldots,\left(E_{n}, 2 j_{n}\right),(E, 2 j)\right)$ is also a special sequence.

(ii) (Tree-like property) If $\left(E_{i}, 2 j_{i}\right)_{i=1}^{n}$ and $\left(F_{l}, 2 k_{l}\right)_{l=1}^{m}$ are both special sequences, then either $j_{i} \neq k_{l}$ for all $i=1, \ldots, n$ and $l=1, \ldots, m$ or there exists a $d \leq \min \{n, m\}$ such that $\left(E_{i}, 2 j_{i}\right)=\left(F_{i}, 2 k_{i}\right)$ for all $i \leq d-1$, $j_{d}=k_{d}$, but $E_{d} \neq F_{d}$ and $j_{i} \neq k_{i}$ for all $d+1 \leq i \leq \min \{n, m\}$.

It is not difficult to see that for each $j \in \mathbb{N}$, each $\|\cdot\|_{j}$ is equivalent to the original norm $\|\cdot\|$. The behavior of the odd indexed norm is the most critical in prescribing 
the asymmetric properties of the space. In particular, the norms $\|\cdot\|_{2 j+1}$ exhibit the following seemingly contradictive behavior. In every block subspace, on one hand, we can find a normalized sequence $\left(x_{k}\right)_{k=1}^{d}$, a special sequence $\left(E_{k}, 2 j_{k}\right)_{k=1}^{d}$ and a $\left(b_{k}\right)_{k=1}^{d} \in B a\left(\ell_{2}\right)$ satisfying: $\left(\operatorname{maxsupp} x_{k}\right)_{k=1}^{d},\left(\min E_{k}\right)_{k=1}^{d} \in S_{n_{2 j+1}},\left\|x_{k}\right\|_{2 j_{k}}=1$, $k=1, \ldots, d, \operatorname{supp} x_{k} \cap E_{k}=\emptyset$ for all $k=1, \ldots, d$ and

$$
\left\|\sum_{k=1}^{d} b_{k} x_{k}\right\| \leq \frac{C}{m_{2 j+1}^{2}}
$$

for some universal constant $C \geq 1$. On the other hand, we may also find $\left(y_{k}\right)_{k=1}^{d}$ such that $\operatorname{supp} y_{k} \subset E_{k}$ and

$$
\left\|\sum_{k=1}^{d} b_{k} y_{k}\right\| \geq \frac{\theta}{m_{2 j+1}},
$$

for some predetermined $\theta>0$. The fact that these estimates differ by a factor of $m_{2 j+1}$ is the critical point, and their existence in every block subspace yields the fundamental properties for the operators.

This work includes the crystallization of the methods for evaluating norms in the fully modified mixed Tsirelson setting. It is important to note that in this case the basic inequality, an important ingredient in previous constructions which reduces the complexity, is not, and perhaps cannot be, used. Some of our techniques can be traced to earlier papers $([1,5,6,[1,, 15])$. In addition to the complexity inherit in dealing with the modified structure, complications arise related to the asymptotic $\ell_{2}$ structure. One should take note that our lemmas consider more global estimates on the special convex combinations as opposed to pointwise estimates found in the asymptotic $\ell_{1}$ cases.

To conclude we state two important open problems in this area. A remarkable result of N. Tomczak-Jaegermann and R. A. Komorowski [21] implies that our space $\mathfrak{X}_{w h}$, as well as the 2-convexification of modified Tsirelson space, contains a (necessary weak Hilbert) subspace without an unconditional basis. The next question was posed by P. Casazza and can be found in [2].

Problem 1. Does there exist a weak Hilbert space which does not embed into a space with an unconditional basis?

Finally, the ultimate problem concerning the existence of singular weak Hilbert spaces was also posed by P. Casazza.

Problem 2. Does there exist a hereditarily indecomposable weak Hilbert space?

\section{SCHREIER FAMILIES AND REPEATED AVERAGES}

In this section we recall the definition of the Schreier families and their modified versions. We also give the definition of $p$-special convex combinations and use the Repeated Averages Hierarchy, introduced in [8], to prove their existence.

The recursive definition of the generalized Schreier hierarchy $\left(\mathcal{S}_{n}\right)_{n<\omega}$ is as follows.

Definition 3.1. Set $\mathcal{S}_{1}=\{F \subset \mathbb{N}:|F| \leq \min F\}$. Let $n \in \mathbb{N}$ and suppose that $\mathcal{S}_{n}$ has been defined. We set $\mathcal{S}_{n+1}=\left\{\bigcup_{i=1}^{d} F_{i}: F_{1}<\ldots<F_{d}, F_{i} \in \mathcal{S}_{n}\right.$, for $1 \leq i \leq$ $\left.d,\left\{\min F_{i}\right\}_{i=1}^{d} \in \mathcal{S}_{1}\right\}$. 
Note that for all $n \in \mathbb{N}$ the family $\mathcal{S}_{n}$ is compact, hereditary and spreading. We also use the following notation for the convolution of two compact, hereditary and spreading families of finite subsets of $\mathbb{N}$.

Notation 3.2. Let $Q, P$ be two families of finite subsets of $\mathbb{N}$. We denote by $P[Q]$ the following family:

$$
P[Q]=\left\{\bigcup_{i=1}^{d} F_{i}: F_{1}<\ldots<F_{d}, F_{i} \in Q \text { for } 1 \leq i \leq d,\left\{\min F_{i}\right\}_{i=1}^{d} \in P\right\} .
$$

Observe that $\mathcal{S}_{n+1}=\mathcal{S}_{1}\left[\mathcal{S}_{n}\right]$ and that, more generally, $\mathcal{S}_{l+k}=\mathcal{S}_{l}\left[\mathcal{S}_{k}\right]=\mathcal{S}_{k}\left[\mathcal{S}_{l}\right]$, for all $k, l \in \mathbb{N}$. Throughout this article we will be using the modified versions of the generalized Schreier families. These are defined as follows:

Definition 3.3. Set $\mathcal{S}_{1}^{M}=\mathcal{S}_{1}$. Let $n \in \mathbb{N}$ and suppose that $\mathcal{S}_{n}^{M}$ has been defined. We set $\mathcal{S}_{n+1}^{M}=\left\{\bigcup_{i=1}^{d} F_{i}:\left(F_{i}\right)_{i=1}^{d}\right.$ are pairwise disjoint, $F_{i} \in \mathcal{S}_{n}^{M}$, for $1 \leq i \leq$ $\left.d,\left\{\min F_{i}\right\}_{i=1}^{d} \in \mathcal{S}_{1}\right\}$.

The analogous notation for the modified convolution is the following.

Notation 3.4. Let $Q, P$ be two families of finite subsets of $\mathbb{N}$. We denote by $P[Q]_{M}$ the following family:

$$
\begin{aligned}
& P[Q]_{M}=\left\{\bigcup_{i=1}^{d} F_{i}:\left(F_{i}\right)_{i=1}^{d}\right. \text { are pairwise disjoint, } \\
& \left.\qquad F_{i} \in Q \text { for } 1 \leq i \leq d,\left\{\min F_{i}\right\}_{i=1}^{d} \in P\right\} .
\end{aligned}
$$

Again observe that the definition of the $\left(\mathcal{S}_{n}^{M}\right)_{n<\omega}$ is equivalent to setting $\mathcal{S}_{n+1}^{M}=$ $\mathcal{S}_{1}^{M}\left[\mathcal{S}_{n}^{M}\right]_{M}$ and that more generally $\mathcal{S}_{l+k}^{M}=\mathcal{S}_{l}^{M}\left[\mathcal{S}_{k}^{M}\right]_{M}=\mathcal{S}_{k}^{M}\left[\mathcal{S}_{l}^{M}\right]_{M}$, for all $k, l \in \mathbb{N}$. It is proved in [5] that for all $n \in \mathbb{N}$ we have $\mathcal{S}_{n}=\mathcal{S}_{n}^{M}$.

For $E_{i} \subset \mathbb{N}$ we say $\left(E_{i}\right)_{i=1}^{d}$ is $S_{n}$-admissible if $E_{1}<E_{2}<\ldots<E_{d}$ and $\left(\min E_{i}\right)_{i=1}^{d} \in S_{n}$. A sequence of finite sets of $\mathbb{N},\left(E_{i}\right)_{i=1}^{d}$, is $S_{n}$-allowable if $\left(E_{i}\right)_{i=1}^{d}$ is pairwise disjoint and $\left(\min E_{i}\right)_{i=1}^{d} \in S_{n}$. A sequence of vectors $\left(x_{i}\right)_{i=1}^{d}$ is $S_{n^{-}}$ allowable (resp. admissible) if ( $\left.\operatorname{supp} x_{i}\right)_{i=1}^{d}$ is $S_{n}$-allowable (resp. admissible).

We are ready to define the $p$-special convex combinations.

Definition 3.5. Let $n \in \mathbb{N}, F \in \mathcal{S}_{n}, p \geq 1, \varepsilon>0$ and $x=\sum_{k \in F} b_{k} e_{k} \in c_{00}$. The vector $x$ will be called a $(p, \varepsilon, n)$-basic special convex combination (bscc) if the following hold:

(1) $\left(\sum_{k \in F} b_{k}^{p}\right)^{1 / p}=1$.

(2) For all $k<n$ and $G \in \mathcal{S}_{k},\left(\sum_{k \in G} b_{k}^{p}\right)^{1 / p}<\varepsilon$.

Definition 3.6. Let $n \in \mathbb{N}, F \in \mathcal{S}_{n}, p \geq 1, \varepsilon>0$ and $x=\sum_{k=1}^{d} b_{k} y_{k}$, such that $\left(y_{k}\right)_{k=1}^{d}$ a block sequence in $c_{00}$. The vector $x$ will be called a $(p, \varepsilon, n)$-special convex combination (scc) if $\sum_{k=1}^{d} b_{k} e_{t_{k}}$ is a $(p, \varepsilon, n)$-bscc, where $t_{k}=\operatorname{maxsupp} y_{k}$, for all $k \in\{1, \ldots, d\}$. 
Remark 3.7. If $\sum_{k=1}^{d} b_{k} y_{k}$ is a $(p, \varepsilon, n)$-scc, then the following hold:

(i) $\left\{\operatorname{maxsupp} y_{k}\right\}_{k=1}^{d} \in \mathcal{S}_{n}$.

(ii) For each $l<n$ and $G \subset\{1, \ldots, d\}$ such that $\left\{\operatorname{maxsupp} y_{k}: k \in G\right\} \in \mathcal{S}_{l}$, $\left(\sum_{k \in G} b_{k}^{p}\right)^{1 / p}<\varepsilon$

For the sake of completeness we prove the existence of the averages defined above. Similar averages have been used in [1] and [1]. We follow the notation of [9].

Definition 3.8. Let $L \in[\mathbb{N}]$. The $n$-averages, $\left(a_{n}^{L}\right)_{n} \in c_{00}$, are defined recursively as follows. For $n=0$ we set $a_{0}^{L}=e_{\min L}$. Suppose we have defined $a_{n}^{M}$ for some $n \in \mathbb{N}$ and all $M \in[\mathbb{N}]$. Then set $l_{1}=\min L, L_{1}=L$ and $L_{k}=L_{k-1} \backslash \operatorname{supp} a_{n}^{L_{k-1}}$, for $k=2, \ldots, l_{1}$. Finally,

$$
a_{n+1}^{L}=\frac{1}{l_{1}}\left(a_{n}^{L_{1}}+\ldots+a_{n}^{L_{l_{1}}}\right) .
$$

The following properties of the RAA can be easily verified using induction:

(1) $\left\|a_{n}^{L}\right\|_{1}=1$ for all $n \in \mathbb{N}$ and $L \in[\mathbb{N}]$, and $a_{n}^{L}(k) \geq 0$ for all $k \in \mathbb{N}$.

(2) $\operatorname{supp} a_{n}^{L}$ is the (unique) maximal initial segment of $L$ that belongs to $\mathcal{S}_{n}$.

The following proposition establishes the presence of a $(1, \varepsilon, n)$-bscc in $c_{00}(\mathbb{N})$ for all $n \in \mathbb{N}$ and $\epsilon>0$.

Proposition 3.9. Let $n \in \mathbb{N}, L \in[\mathbb{N}]$. For all $m<n$ and $G \in \mathcal{S}_{m}$ we have that

$$
\sum_{k \in G} a_{n}^{L}(k)<\frac{3}{\min L} .
$$

We refer the interested reader to 9 for a detailed proof of the above. The existence of a $p$-bscc, for $p>1$, is an immediate consequence of the following.

Remark 3.10. Let $\varepsilon>0, n \in \mathbb{N}, F \in \mathcal{S}_{n}$ and $p>1$. If $x=\sum_{k \in F} b_{k} e_{k}$ is a $\left(1, \varepsilon^{p}, n\right)$-bscc, then $y=\sum_{k \in F} b_{k}^{1 / p} e_{k}$ is a $(p, \varepsilon, n)$-bscc.

Using Proposition 3.9 and Remark 3.10 we can readily establish the following.

Remark 3.11. Let $\left(y_{k}\right)_{k=1}^{\infty}$ be a block sequence in $c_{00}, \varepsilon>0$ and $n \in \mathbb{N}$. There is an interval $E \subset \mathbb{N}$ and $\left(b_{k}\right)_{k \in E}$ such that $\sum_{k \in E} b_{k} y_{k}$ is a $(\varepsilon, n)$-scc. Moreover, if $3 / \operatorname{maxsupp} y_{1}<\varepsilon$ and $d \in \mathbb{N}$ is such that $\left\{\operatorname{maxsupp} y_{k}\right\}_{k=1}^{d}$ is a maximal element of $\mathcal{S}_{n}$, then there exists $\left(b_{k}\right)_{k=1}^{d} \in c_{00}$ such that $\sum_{k=1}^{d} b_{k} y_{k}$ is a $(\varepsilon, n)$-scc.

In the sequel we work only with the case $p=2$, so whenever we consider a $(2, \epsilon, n)$-scc, for some $\epsilon>0$ and $n \in \mathbb{N}$, we shall refer to it as an $(\epsilon, n)$-scc.

\section{The Definition of $\mathfrak{X}_{w h}$ AND its Basic properties}

Definition 4.1. Let $D \subset c_{00}, m>1$ and $n \in \mathbb{N}$. We say that $D$ is closed in the modified $\ell_{2}-\left(1 / m, S_{n}\right)$ operation if for every $\left(f_{i}\right)_{i=1}^{d} \subset D$ such that $\left(f_{i}\right)_{i=1}^{d}$ is $S_{n}$-allowable and $\left(\lambda_{i}\right)_{i=1}^{d} \in B a\left(\ell_{2}\right)$, the vector

$$
\frac{1}{m} \sum_{i=1}^{d} \lambda_{i} f_{i} \in D .
$$

Let $\omega(f)=m$ (weight of $f$ ) when $f$ is the result of the above operation. 
The definition of the space $\mathfrak{X}_{w h}$ requires that we fix two increasing sequences of positive integers $\left(n_{i}\right)_{i=0}^{\infty}$ and $\left(m_{i}\right)_{i=0}^{\infty}$ satisfying certain growth conditions. Let $m_{0}=m_{1}=2, n_{0}=1$, and for $j \geq 2$ let:

(1) $m_{j+1} \geq m_{j}^{3}, \ell_{j}=3 \log _{2}\left(m_{j}\right)+1$.

(2) $n_{j}$ is chosen such that $\ell_{j}\left(n_{j-1}+1\right)<n_{j}$.

As in Section 2, let $N_{1}$ and $N_{2}$ be infinite subsets of $\mathbb{N}$ with $\mathbb{N}=N_{1} \cup N_{2}$ and let

$$
\begin{aligned}
\Sigma=\left\{\left(E_{i}, 2 j_{i}\right)_{i=1}^{n}:\right. & E_{i} \cap E_{j}=\emptyset \text { and } j_{1}<j_{2}<\ldots<j_{n} \\
& \text { with } \left.j_{1} \in N_{1} \text { and } j_{i} \in N_{2} \text { for } i>1\right\} .
\end{aligned}
$$

Define an injective coding $\sigma: \Sigma \rightarrow N_{2}$ such that

$$
m_{2 \sigma\left(\left(E_{1}, 2 j_{1}\right), \ldots,\left(E_{i+1}, 2 j_{i+1}\right)\right)}>m_{2 \sigma\left(\left(E_{1}, 2 j_{1}\right), \ldots,\left(E_{i}, 2 j_{i}\right)\right)} \cdot\left(\operatorname{maxsupp} E_{i}\right)^{2} .
$$

Definition 4.2 ( $\sigma$-special sequences).

(1) A sequence $\left(E_{i}, 2 j_{i}\right)_{i=1}^{\infty}$ is $\sigma$-special if $j_{1} \in N_{1}$, and for each $i \geq 1$,

$$
\sigma\left(\left(E_{1}, 2 j_{1}\right), \ldots,\left(E_{i}, 2 j_{i}\right)\right)=j_{i+1} .
$$

(2) A $\sigma$-special sequence $\left(E_{i}, 2 j_{i}\right)_{i=1}^{p}$ is an $S_{n_{2 j+1}} \sigma$-special sequence if $\left(\min E_{i}\right)_{i=1}^{p} \in S_{n_{2 j+1}}$ and $2 j_{1}>2 j+2$.

(3) $\left(f_{i}\right)_{i=1}^{p} \subset c_{00}\left(\mathbb{N}\right.$ ) is a $\sigma$-special sequence of functionals (an $S_{n_{2 j+1}} \sigma$-special sequence of functionals) if there exists a $\sigma$-special sequence $\left(E_{i}, 2 j_{i}\right)_{i=1}^{p}$ (an $S_{n_{2 j+1}} \sigma$-special sequence resp.) such that $\operatorname{supp} g_{i} \subset E_{i}$ and $\omega\left(f_{i}\right)=m_{2 j_{i}}$ for each $1 \leq i \leq p$.

It follows from the definition that the $\sigma$-special sequences satisfy the extension property and the tree-like property from Section 2.

Definition 4.3. The norming set $D_{w h}$ is the minimal subset of $c_{00}(\mathbb{N})$ such that:

(1) $D_{w h}$ contains $\left\{ \pm e_{n}^{*}: n \in \mathbb{N}\right\}$.

(2) $D_{w h}$ is closed under $\ell_{2}-\left(1 / m_{2 j}, S_{n_{2 j}}\right)$ operations.

(3) $D_{w h}$ contains $f=1 / m_{2 j+1} \sum_{i=1}^{p} \lambda_{i} f_{i}$ such that $\left(\lambda_{i}\right)_{i=1}^{p} \in B a\left(\ell_{2}\right)$ and $\left(f_{i}\right)_{i=1}^{p}$ is an $S_{n_{2 j+1}} \sigma$-special sequence of functionals.

The next definition concerns a decomposition, or tree analysis, of each $f \in D_{w h}$. It is routine to check that every $f \in D_{w h}$ admits such an analysis.

Definition 4.4 (Tree analysis). Let $f \in D_{w h}$. A tree analysis of $f$ is a set $\left(f_{\alpha}\right)_{a \in \mathcal{A}}$ such that $\mathcal{A}$ is a finite tree with a unique root $0 \in \mathcal{A}$ satisfying the following conditions:

(1) $f_{0}=f$ and $f_{\alpha} \in D$ for all $\alpha \in \mathcal{A}$.

(2) $\alpha \in \mathcal{A}$ is terminal if and only if $f_{\alpha} \in D_{0}$.

(3) Let $\alpha \in \mathcal{A}$ be non-terminal. Denote by $S_{\alpha}$ the set of all immediate successors of $\alpha$ in $\mathcal{A}$. Then there exists $j \in \mathbb{N}$ and $\sum_{\beta \in S_{\alpha}} \lambda_{\beta}^{2} \leq 1$ such that

$$
f_{\alpha}=\frac{1}{m_{j}} \sum_{\beta \in S_{\alpha}} \lambda_{\beta} f_{\beta}
$$

If $j$ is even, $\left(f_{\beta}\right)_{\beta \in S_{\alpha}}$ is $S_{n_{j}}$-allowable, and if $j$ is odd, $\left(f_{\beta}\right)_{\beta \in S_{\alpha}}$ is an $S_{n_{2 j+1}}$ $\sigma$-special sequence of functionals. 
Notation 4.5. Let $f \in D_{w h}$ and fix a tree analysis $\left(f_{\alpha}\right)_{\alpha \in \mathcal{A}}$ of $f$.

(1) Let $m(\alpha)=\Pi_{\beta \prec \alpha} \omega\left(f_{\beta}\right)$ for $\alpha \neq 0$.

(2) Let $\lambda(\alpha)=\Pi_{\beta \preceq \alpha} \lambda_{\beta}$ for $\alpha \neq 0$.

Definition 4.6 (The tree representation). Let $\left(f_{\ell}\right)_{\ell=1}^{r} \subset D_{w h}$ and $\left(\lambda_{\ell}\right)_{\ell=1}^{r} \in$ $B a\left(\ell_{2}\right)$. For each $\ell \in\{1, \ldots, r\}$ let $\left(f_{\alpha}\right)_{\alpha \in \mathcal{A}_{\ell}}$ be the tree analysis of $f_{\ell}$ and let $\ell \in \mathcal{A}_{\ell}$ denote its unique root. Set $\mathcal{A}=\bigcup_{\ell=1}^{r} \mathcal{A}_{\ell}$. The collection $\left(f_{\alpha}\right)_{\alpha \in \mathcal{A}}$ is a tree representation of $\sum_{\ell=1}^{r} \lambda_{\ell} f_{\ell}$.

Notation 4.7. For each $\alpha \in \mathcal{A}$ we let:

(1) $\lambda(\alpha)=\lambda_{\ell} \prod_{\gamma \prec \alpha} \lambda_{\gamma}$.

(2) $m(\alpha)=\prod_{\gamma \prec \alpha} \omega\left(f_{\gamma}\right)$.

In the following remark we state some basic properties concerning antichains of $\mathcal{A}$ where $\left(f_{a}\right)_{a \in \mathcal{A}}$ is a tree analysis of a functional $f$ or a tree representation of a functional of the form $\sum_{\ell=1}^{r} \lambda_{\ell} f_{\ell}$.

Remark 4.8. Let $\left(f_{a}\right)_{a \in \mathcal{A}}$ be a tree analysis or a tree representation. If $D \subset \mathcal{A}$ is an antichain, then the following can be readily verified:

1. $\sum_{\gamma \in D} \lambda^{2}(\gamma) \leq 1$.

2. If $D$ is maximal, then $f=\sum_{\gamma \in D} \frac{\lambda(\gamma)}{m(\gamma)} f_{\gamma}$.

3. For $x \in \mathfrak{X}_{w h}$, if we set $x^{D}=\left.x\right|_{\cup_{\gamma \in D}} \operatorname{supp} f_{\gamma}$, then,

$$
f\left(x^{D}\right)=\left.\sum_{\gamma \in D} \frac{\lambda(\gamma)}{m(\gamma)} f_{\gamma}\right|_{\operatorname{supp} x^{D}}\left(x^{D}\right) .
$$

At this point we state the following result which concerns the allowability of families of functionals defined through antichains of a given tree representation.

Lemma 4.9. Let $j \in \mathbb{N},\left(f_{\ell}\right)_{\ell=1}^{r} \subset D_{w h}$ be an $S_{n_{j-1}}$-allowable family and $\left(f_{a}\right)_{a \in \mathcal{A}}$ be a tree representation of the family $\left(f_{\ell}\right)_{\ell=1}^{r} \subset D_{w h}$ and

$$
F=\left\{a \in \mathcal{A}: \prod_{\beta \prec a} \frac{1}{w\left(f_{\beta}\right)}>\frac{1}{m_{j}^{3}}, w\left(f_{\beta}\right) \leq m_{j-1} \text { for all } \beta \prec a\right\} .
$$

Then for every antichain $G \subseteq F$ the family $\left\{f_{a}: a \in G\right\}$ is $S_{n_{j}-1}$-allowable.

Proof. Let $a \in F$. It is easily verified that $|\{\beta \in \mathcal{A}: \beta \prec a\}|<3 \log _{2}\left(m_{j}\right)$. The convolution property of the modified Schreier families and the fact that for all $\beta \prec a$ $w\left(f_{\beta}\right) \leq m_{j-1}$ yield that for a given $\ell \in\{1, \ldots, r\}$ the family $\left\{f_{a}: a \in F \cap \mathcal{A}_{\ell}\right\}$ is


result.

For the definition of $\mathfrak{X}_{w h}$ it is now routine to check that the norm satisfies the implicit formulas stated in the introduction. We restate the norms here for reference. Note that $\mathfrak{X}_{w h}$ is the completion of $c_{00}$ in the norm

$$
\|x\|=\max \left\{\sup \left\{\|x\|_{j}: j \in \mathbb{N} \cup\{0\}\right\},\|x\|_{\infty}\right\},
$$


where for each $j \in \mathbb{N},\|\cdot\|_{j}$ satisfy the following implicit formulas:

(3)

$$
\begin{aligned}
& \|x\|_{2 j}=\sup \left\{\frac{1}{m_{2 j}}\left(\sum_{i=1}^{k}\left\|E_{i} x\right\|^{2}\right)^{\frac{1}{2}}:\left(E_{i}\right)_{i=1}^{k} \text { is } S_{n_{2 j}} \text {-allowable }\right\}, \\
& \|x\|_{2 j+1}=\sup \left\{\frac{1}{m_{2 j+1}}\left(\sum_{i=1}^{k}\left\|E_{i} x\right\|_{2 j_{i}}^{2}\right)^{\frac{1}{2}}:\left(E_{i}, 2 j_{i}\right)_{i=1}^{k} \text { is an } S_{n_{2 j+1}}-\sigma \text { special sequence }\right\} .
\end{aligned}
$$

We now establish the following:

Proposition 4.10. The basis $\left(e_{i}\right)_{i \in \mathbb{N}}$ of $\mathfrak{X}_{w h}$ is asymptotic $\ell_{2}$ for vectors with disjoint support, and therefore $\mathfrak{X}_{w h}$ is a weak Hilbert space.

The proof of this proposition follows from Remark 4.11 and Lemma 4.13. The next remark follows from equation (3).

Remark 4.11. For every sequence of disjointly supported vectors $\left(x_{k}\right)_{k=1}^{d}$ such that $d \leq \operatorname{supp} x_{i}$ for $i \in\{1, \ldots, d\}$,

$$
\frac{1}{2}\left(\sum_{k=1}^{d}\left\|x_{k}\right\|^{2}\right)^{\frac{1}{2}} \leq\left\|\sum_{k=1}^{d} x_{k}\right\| .
$$

The following remark is a critical, however simple, observation we use in the proof of Lemma 4.13

Remark 4.12. Let $f=1 / m_{j} \sum_{i=1}^{p} a_{i} f_{i} \in D_{w h}$ and $x \in \mathfrak{X}_{w h}$. Then there is a $g \in D_{w h}$ such that supp $g \subseteq \operatorname{supp} f$ and

$$
g(x)=\frac{1}{m_{j}}\left(\sum_{i=1}^{p}\left|f_{i}(x)\right|^{2}\right)^{\frac{1}{2}} .
$$

Proof. If $f_{i}(x)=0$ for all $i \in\{1, \ldots, d\}$, let $f=g$. Otherwise observe that

$$
g=\frac{1}{m_{j}} \sum_{i=1}^{d} \frac{f_{i}(x)}{\left(\sum_{j=1}^{d} f_{j}(x)^{2}\right)^{\frac{1}{2}}} f_{i}
$$

does the trick.

Lemma 4.13. Let $f \in D_{w h}$ and $\left(x_{k}\right)_{k=1}^{d}$ be a sequence of disjointly supported vectors. There exists a disjointly supported sequence $\left(g_{k}\right)_{k=1}^{d} \subset D_{w h}$ with $\operatorname{supp} g_{k} \subset$ $\operatorname{supp} f$ for all $k \in\{1, \ldots, d\}$ such that

$$
f\left(\sum_{k=1}^{d} x_{k}\right) \leq\left(\sum_{k=1}^{d}\left|g_{k}\left(x_{k}\right)\right|^{2}\right)^{\frac{1}{2}} .
$$

Moreover, the standard basis of $\mathfrak{X}_{w h}$ satisfies an upper $\ell_{2}$ estimate on vectors with disjoint support.

Proof. The "moreover" statement follows immediately from the first conclusion since $\left|g_{k}\left(x_{k}\right)\right| \leq\left\|x_{k}\right\|$. We proceed by induction on the height of the tree analysis of $f \in D_{w h}$. If $f= \pm e_{n}^{*}$ the claim is obvious. Assume the conclusion holds for all $k<n$ and let $f$ have a tree analysis of height $n$ with $f=1 / m_{j} \sum_{i=1}^{p} \lambda_{i} f_{i}$. 
By applying the induction hypothesis for each $f_{i}$ and then the Cauchy-Schwartz inequality, we have

$$
\frac{1}{m_{j}} \sum_{i=1}^{p} \lambda_{i} f_{i}\left(\sum_{k=1}^{d} x_{k}\right) \leq \sum_{i=1}^{p} \lambda_{i}\left(\sum_{k=1}^{d}\left|g_{k, i}\left(x_{k}\right)\right|^{2}\right)^{\frac{1}{2}} \leq \frac{1}{m_{j}}\left(\sum_{k=1}^{d} \sum_{i=1}^{p}\left|g_{k, i}\left(x_{k}\right)\right|^{2}\right)^{\frac{1}{2}} .
$$

For each $k \in\{1, \ldots, d\}$ and any $\left(a_{i}\right)_{i=1}^{p} \in B a\left(\ell_{2}\right), 1 / m_{j} \sum_{i=1}^{d} a_{i} g_{k, i} \in D_{w h}$. Now apply Remark 4.12 for each $k=1, \ldots, d$ to find $g_{k} \in D$ with $\operatorname{supp} g_{k} \subset$ $\bigcup_{i=1}^{d} \operatorname{supp} g_{k, i}$ such that

$$
\left|g_{k}\left(x_{k}\right)\right| \leq\left(\sum_{i=1}^{n}\left|g_{k, i}\left(x_{k}\right)\right|^{2}\right)^{1 / 2}
$$

The result follows.

\section{RAPIDLY INCREASING SEQUENCES, EXACT VECTORS AND 0-DEPENDENT SEQUENCES}

In this section we state relevant definitions that lay the groundwork for Section 7 where we prove that the operators have the desired decomposition. The following definition is a fundamental component in the construction of all spaces with few operators.

Definition 5.1. Let $C>0$ and $\left(2 j_{k}\right)_{k}$ be an increasing sequence of positive integers. A block sequence $\left(x_{k}\right)_{k \in \mathbb{N}}$ in $\mathfrak{X}_{w h}$ is a $\left(C,\left(2 j_{k}\right)_{k}\right)$-rapidly increasing sequence (RIS) if the following are satisfied for each $k$ :

(1) $\left\|x_{k}\right\| \leq C$.

(2) Each $x_{k}$ is a $\left(1 / m_{2 j_{k}}^{3}, n_{2 j_{k}}\right)$-scc.

(3) $\left(\operatorname{maxsupp} x_{k}\right)^{2} / m_{2 j_{k+1}}<1 / m_{2 j_{k}}$.

Definition 5.2. A block sequence $\left(x_{k}\right)_{k \in \mathbb{N}}$ is a seminormalized $\left(C,\left(2 j_{k}\right)_{k=1}^{\infty}\right)$-RIS if it is a $\left(C,\left(2 j_{k}\right)\right)$-RIS and $\left\|x_{k}\right\| \geq 1$ for all $k \in \mathbb{N}$.

Finding an RIS is done inductively. The next lemma establishes the existence of seminormalized scc's in every block subspace.

Lemma 5.3. Let $\left(x_{k}\right)$ be a normalized block sequence in $\mathfrak{X}_{w h}, \varepsilon>0$ and $j \geq 2$. Then there exists a block sequence $\left(y_{k}\right)_{k}$ of $\left(x_{k}\right)_{k}$ such that $\left\|y_{k}\right\| \leq 1$ and $a\left(\varepsilon, n_{2 j}\right)$ scc $\sum_{k=1}^{d} a_{k} y_{k}$ such that $\left\|\sum_{k=1}^{d} a_{k} y_{k}\right\| \geq 1 / 2$.

The proof of the preceding lemma is identical to Lemma 4.5 in [6], making the obvious modifications to accommodate the $\ell_{2}$ structure. The next definition is new.

Definition 5.4 (Exact vector). Let $\left(x_{k}\right)_{k \in \mathbb{N}}$ be a seminormalized $\left(C,\left(2 j_{k}\right)_{k=1}^{\infty}\right)$ RIS. A vector $x=m_{2 j} \sum_{k \in E} b_{k} x_{k}$ is a $(C, 2 j)$ exact vector with respect to $\left(x_{k}\right)_{k \in \mathbb{N}}$ if $j<j_{\min E}$ and $\sum_{k \in E} b_{k} x_{k}$ is a $\left(1 / m_{2 j}^{3}, n_{2 j}\right)$-scc.

Definition 5.5 (Exact sequence). Let $\left(y_{s}\right)$ be a seminormalized $\left(C,\left(2 i_{s}\right)\right)$-RIS. A block sequence $\left(x_{k}\right)$ is a $\left(C,\left(2 j_{k}\right)\right)$ exact sequence with respect to $\left(y_{s}\right)$ if:

(1) Each $x_{k}$ is a $\left(C, 2 j_{k}\right)$ exact vector with respect to $\left(y_{s}\right)$.

(2) $\operatorname{minsupp} x_{k} \leq \operatorname{minsupp} y_{s}$ implies $j_{k}<i_{s}$.

(3) $\operatorname{maxsupp} y_{s}<\operatorname{minsupp} x_{k}$ implies $j_{k}>i_{s}$.

A block sequence $\left(x_{k}\right)_{k \in \mathbb{N}}$ is a $\left(C,\left(2 j_{k}\right)\right)$ exact sequence if it is an exact sequence with respect to some RIS. 
The existence of such a sequence in every block subspace is straightforward.

Next we define the crucial notion that allows us to establish the desired decomposition of operators on $\mathfrak{X}_{w h}$.

Definition 5.6. Let $j \in \mathbb{N}, C>1$. A block sequence $\left(x_{k}\right)_{k \in \mathbb{N}}$ is a $(0, C, 2 j+1)$ dependent sequence if:

(1) $\left(x_{k}\right)$ is a $\left(C,\left(2 j_{k}\right)\right)$ exact sequence.

(2) $j+1<j_{1} \in N_{1}$, and there is a sequence $\left(E_{i}\right)_{i=1}^{\infty}$ such that $\left(E_{i}, 2 j_{i}\right)_{i=1}^{\infty}$ is $\sigma$ special with $\left(\bigcup_{i \in \mathbb{N}} E_{i}\right) \cap\left(\bigcup_{i \in \mathbb{N}} \operatorname{supp} x_{i}\right)=\emptyset$ and $\operatorname{maxsupp} x_{i}<\operatorname{maxsupp} E_{i}$ for all $i \in \mathbb{N}$.

In this case we say that $\left(x_{k}\right)$ is a $(0, C, 2 j+1)$ dependent sequence with respect to $\left(E_{i}, 2 j_{i}\right)_{i=1}^{\infty}$.

Remark 5.7. If $\left(x_{k}\right)_{k \in \mathbb{N}}$ is a $(0, C, 2 j+1)$ dependent sequence, then $\left(\frac{1}{m_{2 j_{k}}} x_{k}\right)$ is a $\left(C,\left(2 j_{k}\right)\right)$-RIS.

Proof. Conditions (1) and (2) in the definition of RIS are immediate. Since $\sigma\left(\left(E_{i}, 2 j_{i}\right)_{i=1}^{k}\right)=j_{k+1}$ for all $k \in \mathbb{N}$ and

$$
m_{2 j_{k+1}}>m_{2 j_{k}}\left(\operatorname{maxsupp} E_{k}\right)^{2}>m_{2 j_{k}}\left(\operatorname{maxsupp} x_{k}\right)^{2},
$$

condition (3) is verified.

\section{THE BASIC EVALUATION}

The aim of this section is to show the following result:

Proposition 6.1. Let $j \in \mathbb{N}, C>0$, and $\left(x_{k}\right)_{k=1}^{d}$ be a $(0, C, 2 j+1)$ dependent sequence, and let $\sum_{k=1}^{d} b_{k} x_{k}$ be $a\left(1 / m_{2 j+2}^{2}, n_{2 j+1}\right)$-scc and $f \in D_{w h}$ with $w(f)<$ $m_{2 j+1}$. Then

$$
f\left(\sum_{k=1}^{d} b_{k} x_{k}\right) \leq \frac{C}{m_{2 j+1}^{2}} .
$$

In order to prove the above we need some preliminary work.

We begin by providing three fundamental techniques that allow us to derive small estimates.

Lemma 6.2 (Minimum free estimate (MFE)). Suppose $\left(f_{\ell}\right)_{\ell=1}^{p} \subset D_{w h}$ is a sequence of disjoint functionals and $\left(x_{k}\right)_{k=1}^{d}$ is a block sequence in $\mathfrak{X}_{w h}$ with $\left\|x_{k}\right\| \leq C$ such that for all $\ell=1, \ldots, p$ and $k=1, \ldots, d$, minsupp $f_{\ell} \notin \operatorname{ran} x_{k}$. For $\left(\lambda_{\ell}\right)_{\ell=1}^{p} \in$ $B a\left(\ell_{2}\right)$ and $\left(b_{k}\right)_{k=1}^{d} \in c_{00}$,

$$
\sum_{\ell=1}^{p} \lambda_{\ell} f_{\ell}\left(\sum_{k=1}^{d} b_{k} x_{k}\right) \leq 4 C\left(\sum_{k=1}^{d} b_{k}^{2}\right)^{\frac{1}{2}} .
$$

The above lemma, although easy, is critical. It is similar to Lemma 4.9 in [6] and the key point is that it distinguishes the behavior of modified mixed Tsirelson spaces $T_{M}\left[\left(\theta_{n}, \mathcal{S}_{n}\right)_{n}\right]$ built on the Schreier families from the ones of the form $T_{M}\left[\left(\theta_{n}, A_{n}\right)_{n}\right]$ which use the lower complexity families $\left(A_{n}\right)_{n}$. Indeed, as it is known, the latter class (including among others the modified Schlumprecht space) contains members with subspaces isomorphic to $\ell_{1}$ and hence not reflexive (cf. [23]). 
Proof. Let $I_{0}=\emptyset$ and for each $k \in\{1, \ldots, d\}$, define $I_{k}=\left\{\ell: \operatorname{minsupp} f_{\ell}<\right.$ $\left.\operatorname{supp} x_{k}\right\}$. By definition, $\left\{\left.f_{\ell}\right|_{\left[\operatorname{minsupp} x_{k}, \infty\right)}: \ell \in I_{k}\right\}$ is $S_{1}$-allowable. For each $t=$ $1, \ldots, d$, define $\xi_{t}^{2}=\sum_{\ell \in I_{t} \backslash I_{t-1}} \lambda_{\ell}^{2}, g_{t}=\left.(1 / 2) \sum_{\ell \in I_{t} \backslash I_{t-1}}\left(\lambda_{\ell} / \xi_{t}\right) f_{\ell}\right|_{\left[\operatorname{minsupp} x_{k}, \infty\right)} \in$ $D_{w h}$ and $E_{t}=\operatorname{supp} g_{t}$. Applying the upper $\ell_{2}$ estimate, the Cauchy-Schwartz inequality and the the lower $\ell_{2}$ estimate, it follows that,

$$
\begin{gathered}
\sum_{t=1}^{d} \sum_{\ell \in I_{t} \backslash I_{t-1}} \gamma_{\ell} f_{\ell}\left(\sum_{k=1}^{d} b_{k} x_{k}\right)=2 \sum_{t=1}^{d} \xi_{t} g_{t}\left(\sum_{k=t}^{m} b_{k} x_{k}\right) \leq 2 \sum_{t=1}^{d} \xi_{t}\left(\sum_{k=t}^{d}\left\|E_{t}\left(b_{k} x_{k}\right)\right\|^{2}\right)^{\frac{1}{2}} \\
\leq 2\left(\sum_{k=1}^{d} \sum_{t=1}^{k}\left\|E_{t}\left(b_{k} x_{k}\right)\right\|^{2}\right)^{\frac{1}{2}} \leq 4\left(\sum_{k=1}^{d} b_{k}^{2} C^{2}\right)^{\frac{1}{2}} \leq 4 C\left(\sum_{k=1}^{d} b_{k}^{2}\right)^{\frac{1}{2}} .
\end{gathered}
$$

Lemma 6.3 (Small allowability estimate (SAE)). Let $\left(f_{\ell}\right)_{\ell=1}^{r} \subset D_{w h}$ be $S_{q}$ allowable, and $\left(x_{k}\right)_{k=1}^{d}$ a block sequence in $\mathfrak{X}_{w h}$ with $\left\|x_{k}\right\| \leq C$ for $k=1, \ldots, d$ such that $\sum_{k=1}^{d} b_{k} x_{k}$ is an $\left(\epsilon, n_{j_{0}}\right)$-scc with $n_{j_{0}}>q$ and $\epsilon \leq \frac{1}{m_{j_{1}}^{2}}$, where $j_{1}=j_{0}$ if $j_{0}$ is even and $j_{1}=j_{0}+1$ if $j_{0}$ is odd. If we set

$$
\Phi=\left\{k \in\{1, \ldots, d\}: \exists \ell \in\{1, \ldots, r\} \text { with minsupp } f_{\ell} \in \operatorname{ran} x_{k}\right\},
$$

then for $\left(\lambda_{\ell}\right)_{\ell=1}^{r} \in B a\left(\ell_{2}\right)$,

$$
\sum_{\ell=1}^{r} \lambda_{\ell} f_{\ell}\left(\sum_{k=1}^{d} b_{k} x_{k}\right) \leq \frac{C}{m_{j_{0}}} .
$$

Proof. We can readily observe that $\frac{1}{m_{j_{1}}} \sum_{\ell=1}^{r} \lambda_{\ell} f_{\ell} \in D_{w h}$ and also that the family $\left\{\operatorname{maxsupp}_{k}: k \in \Phi\right\}$ is $S_{q}$ allowable. Hence, an application of the upper $\ell_{2^{-}}$ estimate yields

$$
\begin{aligned}
\sum_{\ell=1}^{r} \lambda_{\ell} f_{\ell} & =m_{j_{1}}\left(\frac{1}{m_{j_{1}}} \sum_{\ell=1}^{r} \lambda_{\ell} f_{\ell}\right)\left(\sum_{k \in \Phi} b_{k} x_{k}\right) \\
& \leq C m_{j_{1}}\left(\sum_{k \in \Phi} b_{k}^{2}\right)^{1 / 2} \leq C m_{j_{1}} \cdot \epsilon<\frac{C}{m_{j_{1}}} .
\end{aligned}
$$

Our third and final basic estimate is the one that utilizes the RIS condition.

Lemma 6.4 (RIS estimate (RISE)). Let $\left(x_{k}\right)_{k=1}^{d}$ be a block sequence and $\left(m_{2 j_{k}}\right)_{k=1}^{d+1}$ a sequence of weights such that $\frac{\left(\operatorname{minsupp} x_{k}\right)^{2}}{m_{2 j_{k+1}}} \leq \frac{1}{m_{2 j_{k}}^{2}}$. Then, for every disjoint family $\left(f_{\ell}\right)_{\ell=1}^{r} \subset D_{w h}$ satisfying $\left\|\left.f_{\ell}\right|_{\text {supp } x_{k}}\right\|_{\infty} \leq \frac{1}{m_{2 j_{k+1}}}$ for all $\ell=1, \ldots, r$ and $k=1, \ldots, d$ and $\left(c_{k}\right)_{k=1}^{d} \in c_{00}$, with $0 \leq c_{k} \leq m_{2 j_{k}}$, we have

$$
\sum_{\ell=1}^{r} f_{\ell}\left(\sum_{k=1}^{d} c_{k} x_{k}\right) \leq \frac{2}{m_{2 j_{1}}}
$$

Proof. Let $k \in\{1, \ldots, d\}$. Observe that as the functionals $\left(f_{\ell}\right)_{\ell=1}^{r}$ have disjoint supports, $\left|\left\{\ell: \operatorname{minsupp} f_{\ell} \leq \operatorname{maxsupp} x_{k}\right\}\right| \leq \operatorname{maxsupp} x_{k}$. Hence,

$$
\sum_{\ell=1}^{r} f_{\ell}\left(x_{k}\right) \leq \sum_{\ell=1}^{r}\left\|\left.f_{\ell}\right|_{\operatorname{supp} x_{k}}\right\|_{\infty} \cdot \operatorname{maxsupp} x_{k} \leq \frac{\left(\operatorname{minsupp} x_{k}\right)^{2}}{m_{2 j_{k+1}}} \leq \frac{1}{m_{2 j_{k}}^{2}} .
$$

Summing up for all $k$ yields the result. 
The next lemma concerns that application of an $\ell_{2}$ convex combination of a sequence of functionals with 'small' allowability to a special convex combination of vectors with comparatively large character. The term 'character' refers to the $n$ component of a $(\varepsilon, n)$-scc.

Lemma 6.5. Let $C \geq 1$ and $\left(x_{k}\right)_{k=1}^{d}$ be a block sequence in $\mathfrak{X}_{w h}$ such that $\left\|x_{k}\right\| \leq$ $C$. Let $\sum_{k=1}^{d} b_{k} x_{k}$ be $a\left(\varepsilon, n_{j}\right)$-scc with $\varepsilon \leq 1 / m_{j_{1}}^{2}$, where $j_{1}=j$ if $j$ is even and $j_{1}=j+1$ otherwise. Also let $\left(f_{\ell}\right)_{\ell=1}^{r}$ be $\mathcal{S}_{q}$-allowable for $q<n_{j}$ and $\left(\lambda_{\ell}\right)_{\ell} \in B a\left(\ell_{2}\right)$. Then,

$$
\sum_{\ell=1}^{r} \lambda_{\ell} f_{\ell}\left(\sum_{k=1}^{d} b_{k} x_{k}\right) \leq 5 C
$$

Proof. Let $\Phi=\left\{k \in\{1, \ldots, d\}: \exists \ell \in\{1, \ldots, r\}\right.$ with $\left.\operatorname{minsupp} f_{\ell} \in \operatorname{ran} x_{k}\right\}$ and $\Phi^{C}=\{1, \ldots, k\} \backslash \Phi$. Then,

Applying the SAE (Lemma 6.3) we can see that $\sum_{\ell=1}^{r} \lambda_{\ell} f_{\ell}\left(\sum_{k \in \Phi} b_{k} x_{k}\right) \leq \frac{C m_{j}}{m_{j_{1}}^{2}}$.

By the MFE (Lemma 6.2) $\sum_{\ell=1}^{r} \lambda_{\ell} f_{\ell}\left(\sum_{k \in \Phi^{C}} b_{k} x_{k}\right) \leq 4 C$.

Combining the above yields the result.

The next lemma is more general than the previous in some sense, but is not a direct generalization. Here we assume that the disjoint sequence of functionals is at most $\mathcal{S}_{n_{q}}$-allowable for $n_{q}$ strictly smaller than the characters of a sequence of scc's. In the previous lemma we assumed the allowability was of the sequence of functionals at most one less than the character of one scc. The major difference (or restriction) of course is that the allowability must be indexed by a member of the sequence $\left(n_{i}\right)$, and this is not so in the first lemma. The proof is very similar.

Lemma 6.6. Let $C \geq 1$ and $\left(x_{k}\right)_{k=1}^{d}$ be a block sequence in $\mathfrak{X}_{w h}$ such that $\left\|x_{k}\right\| \leq C$ and each $x_{k}$ is $\left(1 / m_{2 j_{k}}^{2}, n_{2 j_{k}}\right)$-scc and $j_{1} \leq \cdots \leq j_{d}$. Let $\left(f_{\ell}\right)_{\ell=1}^{p} \subset D$ be $\mathcal{S}_{n_{q}{ }^{-}}$ allowable with $q<2 j_{1}$ and $\omega\left(f_{\ell}\right)<m_{2 j_{1}}$. For $\left(\lambda_{\ell}\right)_{\ell=1}^{p} \in B a\left(\ell_{2}\right)$ and $\left(b_{k}\right)_{k=1}^{d} \in c_{00}$,

$$
\sum_{\ell=1}^{p} \lambda_{\ell} f_{\ell}\left(\sum_{k=1}^{d} b_{k} x_{k}\right) \leq \frac{5 C}{\min _{1 \leq \ell \leq p} \omega\left(f_{\ell}\right)}\left(\sum_{k=1}^{d} b_{k}^{2}\right)^{\frac{1}{2}} \text {. }
$$

Proof. For each $k \in\{1, \ldots, d\}$ let $x_{k}=\sum_{1=1}^{p_{k}} b_{k, i} x_{k, i}$. For each $\ell \in\{1, \ldots, p\}$ denote by $S_{\ell}$ the immediate successors of $f_{\ell}$ in its tree analysis and let $S=\bigcup_{\ell=1}^{p} S_{\ell}$. Let

$$
\Phi=\left\{(k, i): \exists \alpha \in S \text { such that } \operatorname{minsupp} f_{\alpha} \in \operatorname{ran} x_{k, i}\right\} .
$$

For each $k \in\{1, \ldots, d\}$ let $\Phi_{k}=\{i:(k, i) \in \Phi\}$. For each $\ell \in\{1, \ldots, p\}$ we have assumed $\left(f_{\alpha}\right)_{\alpha \in S_{\ell}}$ is $\mathcal{S}_{n_{2 j_{1}-1}}$-allowable. By the convolution property of Schreier families we may conclude that $\left\{f_{\alpha}: \alpha \in S\right\}$ is $\mathcal{S}_{2 n_{2 j_{1}-1}}$-allowable. Recall that $2 n_{2 j_{1}-1}<n_{2 j_{1}}$ and observe that $k \in\{1, \ldots, d\}\left\{\operatorname{maxsupp} x_{k, i}: i \in \Phi_{k}\right\} \in \mathcal{S}_{2 n_{2 j_{1}-1}}$. Observe that

$$
\sum_{\ell=1}^{p} \lambda_{\ell} f_{\ell}\left(\sum_{k=1}^{d} b_{k} \sum_{i \in \Phi_{k}} b_{k, i} x_{k, i}\right)=\sum_{\ell=1}^{p} \lambda_{\ell} \frac{1}{\omega\left(f_{\ell}\right)} \sum_{\alpha \in S_{\ell}} \lambda_{\alpha} f_{\alpha}\left(\sum_{k=1}^{d} b_{k} \sum_{i \in \Phi_{k}} b_{k, i} x_{k, i}\right) .
$$

Applying the SAE we obtain

$$
\sum_{\ell=1}^{p} \lambda_{\ell} f_{\ell}\left(\sum_{k=1}^{d} b_{k} \sum_{i \in \Phi_{k}} b_{k, i} x_{k, i}\right) \leq \sum_{k=1}^{d} \frac{C}{m_{2 j_{k}}} \leq \frac{2 C}{m_{2 j_{1}}}
$$


For the remaining part we may apply MFE to see that

$$
\begin{aligned}
\sum_{\ell=1}^{p} \lambda_{\ell} f_{\ell} & \left(\sum_{k=1}^{d} b_{k} \sum_{i \in\left\{1, \ldots, p_{k}\right\} \backslash \Phi_{k}} b_{k, i} x_{k, i}\right) \\
\leq & \frac{1}{\min _{1 \leq \ell \leq p} \omega\left(f_{\ell}\right)} \sum_{\ell=1}^{p} \sum_{\alpha \in S_{\ell}} \lambda_{\ell} \lambda_{\alpha} f_{\alpha}\left(\sum_{k=1}^{d} b_{k} \sum_{i \in\left\{1, \ldots, p_{k}\right\} \backslash \Phi_{k}} b_{k, i} x_{k, i}\right) \\
\leq & \frac{4 C}{\min _{1 \leq \ell \leq p} \omega\left(f_{\ell}\right)} .
\end{aligned}
$$

Combining the above estimates yields the result.

The final element we need in the proof of Proposition 6.1 is the following:

Proposition 6.7. Let $\left(x_{k}\right)_{k=1}^{d}$ be a $\left(C, 2 j_{k}\right)_{k}$ exact sequence and $\left(f_{\ell}\right)_{\ell=1}^{r} \subset D_{w h}$ be $S_{n_{q}}$-allowable with $q<2 j_{1}$. Assume further that for $k \in\{1, \ldots, d\}$ and $\ell \in\{1, \ldots, r\}$ such that $\operatorname{supp} f_{\ell} \cap \operatorname{supp} x_{k} \neq \emptyset$, we have $w\left(f_{\ell}\right) \neq m_{2 j_{k}}$. Then for $\left(\lambda_{\ell}\right)_{\ell=1}^{r},\left(b_{k}\right)_{k=1}^{d} \in$ $B a\left(\ell_{2}\right)$,

$$
\sum_{\ell=1}^{r} \lambda_{\ell} f_{\ell}\left(\sum_{k=1}^{d} b_{k} x_{k}\right) \leq \frac{40 C}{\min _{1 \leq \ell \leq r} w\left(f_{\ell}\right)} .
$$

We shall present the proof of this result in the last section. Granting this we proceed to the proof of Proposition 6.1. Our approach in proving this result is separated into two steps. In the first step, we use the tree analysis $\left(f_{a}\right)_{a \in A}$ of $f$ to split the support of each $x_{k}$ into four disjoint sets and we define the vectors $x_{k}^{G}, x_{k}^{S}, x_{k}^{R_{1}}, x_{k}^{R_{2}}$. The second step is to use the preparatory estimates presented earlier in this section to evaluate the action of $f$ onto each $\sum_{k=1} b_{k} x_{k}^{I}$ where $I$ is one of the $G, S, R_{1}, R_{2}$.

Proof. (Proposition 6.1).

We start by defining the splitting of the support of each $\left(x_{k}\right)$, for $k=1, \ldots, d$.

The partitioning:

Let $k \in\{1, \ldots, d\}$. We define the following sets. $G_{k}=\{t \in \mathcal{A}: t$ is maximal $\left.\operatorname{supp} f_{t} \cap \operatorname{supp} x_{k} \neq \emptyset, \prod_{\gamma \prec t} \frac{1}{w\left(f_{\gamma}\right)} \leq \frac{1}{m_{2 j_{k+1}}}\right\}$.

Let $P_{k}$ denote the maximal $t \in \mathcal{A}$ such that $\operatorname{supp} f_{t} \cap \operatorname{supp} x_{k} \neq \emptyset$ and $t \notin G_{k}$.

For $t \in P_{k}$, let $\beta_{t}=t$ if $w\left(f_{\gamma}\right)<m_{2 j+1}$ for all $\gamma \prec t$ or $\beta_{t}=\min \left\{\gamma \prec t: w\left(f_{\gamma}\right) \geq\right.$ $\left.m_{2 j+1}\right\}$ otherwise.

We set $S_{k}=\left\{\beta_{t}: t \in P_{k}, \frac{1}{m\left(\beta_{t}\right)} \leq \frac{1}{m_{2 j+1}^{3}}\right\}, R_{k}=\left\{\beta_{t}: t \in P_{k}, \frac{1}{m\left(\beta_{t}\right)}>\frac{1}{m_{2 j+1}^{3}}\right\}$.

We split $R_{k}$ into:

$R_{k}^{1}=\left\{\beta_{t} \in R_{k}: w\left(f_{\beta_{t}}\right)=m_{2 j+1}\right\}, R_{k}^{2}=\left\{\beta_{t} \in R_{k}: w\left(f_{\beta_{t}}\right) \geq m_{2 j+2}\right\}$.

Using the above sets we partition $x_{k}$ in the following way: Set $x_{k}^{G}=x_{k} \mid \bigcup_{t \in G_{k}} \operatorname{supp} f_{t}$, $x_{k}^{S}=\left.x_{k}\right|_{\bigcup_{\beta_{t} \in S_{k}}} \operatorname{supp} f_{\beta_{t}}, x_{k}^{R_{1}}=\left.x_{k}\right|_{\bigcup_{\beta_{t} \in R_{k}^{1}} \operatorname{supp} f_{\beta_{t}}}$ and $x_{k}^{R_{2}}=\left.x_{k}\right|_{\bigcup_{\beta_{t} \in R_{k}^{2}} \operatorname{supp} f_{\beta_{t}}}$.

We also set $x^{I}=\sum_{k=1}^{d} b_{k} x_{k}^{I}$, where $I=G, S, R_{1}, R_{2}$, and we denote by $G, S, R_{1}$, $R_{2}$ the union for all $k$ of the corresponding $G_{k}, S_{k}, R_{k}^{1}, R_{k}^{2}$. In the following lemma and corollary we present some easy consequences of the above partitions.

Lemma 6.8. The set $\left\{\beta_{t}: t \in \bigcup_{k=1}^{d} P_{k}\right\}$ is an antichain of $\mathcal{A}$. 
Proof. Let $\beta_{t_{1}} \neq \beta_{t_{2}}$, with $t_{1}, t_{2} \in \bigcup_{i=1}^{d} P_{k}$, and suppose that $\beta_{t_{1}} \prec \beta_{t_{2}}$. As $\beta_{t_{1}}$ is not maximal, $w\left(f_{\beta_{t_{1}}}\right) \geq m_{2 j+1}$. However, by definition, for every $\gamma \prec \beta_{t_{2}}$ we have $w\left(f_{\gamma}\right)<m_{2 j+1}$, which is a contradiction, and the proof is complete.

Combining Lemma 6.8 and Lemma 4.9 we have the following:

Corollary 6.9. For $\beta_{t} \in S$ let $a_{t}=\min \left\{\gamma \prec \beta_{t}: \prod_{\beta \prec \gamma} \frac{1}{w\left(f_{\beta}\right)} \leq \frac{1}{m_{2 j+1}^{2}}\right\}$. Then the families $\left\{f_{a_{t}}: \beta_{t} \in S\right\},\left\{f_{\beta_{t}}: \beta_{t} \in R\right\}$ are $S_{n_{2 j+1}-1}$-allowable.

Let us also observe that

$$
f\left(\sum_{k=1}^{d} b_{k} x_{k}\right)=f\left(\sum_{k=1}^{d} b_{k} x_{k}^{G}\right)+f\left(\sum_{k=1}^{d} b_{k} x_{k}^{S}\right)+f\left(\sum_{k=1}^{d} b_{k} x_{k}^{R_{1}}\right)+f\left(\sum_{k=1}^{d} b_{k} x_{k}^{R_{2}}\right) .
$$

We shall consider the cases given by the partitions separately. We start with $f\left(\sum_{k=1}^{d} b_{k} x_{k}^{G}\right)$. A straightforward application of the RISE (Lemma 6.4) yields

$$
f\left(\sum_{k=1}^{d} b_{k} x_{k}^{G}\right) \leq \frac{2}{m_{2 j_{1}}} \leq \frac{1}{m_{2 j+1}^{2}} .
$$

We now pass to $f\left(\sum_{k=1}^{d} b_{k} x_{k}^{S}\right)$. For $\beta_{t} \in S$ let $a_{t}=\min \left\{\gamma \prec \beta_{t}: \frac{1}{m(\gamma)} \leq \frac{1}{m_{2 j+1}^{2}}\right\}$.

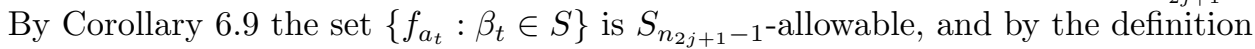
of $\beta_{t}$ we also have that $w\left(f_{a_{t}}\right)<m_{2 j+1}$. Additionally we can readily see that

$$
f\left(\sum_{k=1}^{d} b_{k} x_{k}^{S}\right)=\sum_{\left\{a_{t}: \beta_{t} \in S\right\}} \frac{\lambda\left(a_{t}\right)}{m\left(a_{t}\right)} f_{a_{t}}\left(\sum_{k=1}^{d} b_{k} x_{k}^{S}\right) .
$$

Applying Lemma 6.5 on the sum,

$$
\sum_{\left\{a_{t}: \beta_{t} \in S\right\}} \lambda\left(a_{t}\right) f_{a_{t}}\left(\sum_{k=1}^{d} b_{k} x_{k}^{S}\right) .
$$

We may conclude that

$$
f\left(\sum_{k=1}^{d} b_{k} x_{k}^{S}\right) \leq \frac{1}{m_{2 j+1}^{2}} \sum_{\left\{a_{t}: \beta_{t} \in S\right\}} \lambda\left(a_{t}\right) f_{a_{t}}\left(\sum_{k=1}^{d} b_{k} x_{k}^{S}\right) \leq \frac{5 C}{m_{2 j+1}^{2}} .
$$

We now consider $f\left(\sum_{k=1}^{d} b_{k} x_{k}^{R_{2}}\right)$.

Let $E=\left\{k \leq d: \exists \beta_{t} \in R: \operatorname{supp} f_{\beta_{t}} \cap \operatorname{supp} x_{k}^{R_{2}} \neq \emptyset, w\left(f_{\beta_{t}}\right)=m_{2 j_{k}}\right\}$ and $E^{c}=\{1, \ldots, d\} \backslash E$. Observe that by Corollary 6.9 the family $\left\{\operatorname{maxsupp} x_{k}: k \in\right.$ $E\} \in S_{n_{2 j+1}-1}$. A direct application of the upper $\ell_{2}$ estimate yields

$$
f\left(\sum_{k \in E} b_{k} x_{k}^{R_{2}}\right) \leq C\left(\sum_{k \in E} b_{k}^{2}\right)^{1 / 2} \leq C \frac{1}{m_{2 j+1}^{2}} .
$$

For $k \in E^{c}$ we can see that

$$
f\left(\sum_{k \in E^{c}} b_{k} x_{k}^{R_{2}}\right)=\sum_{\left\{\beta_{t} \in R^{2}\right\}} \frac{\lambda\left(\beta_{t}\right)}{m\left(\beta_{t}\right)} f_{\beta_{t}}\left(\sum_{k \in E^{c}} b_{k} x_{k}^{R_{2}}\right) .
$$

By the definition of the set $E^{c}$ for $\beta_{t} \in R^{2}$ and $k \in E^{c}$ if $\operatorname{supp} f_{\beta_{t}} \cap \operatorname{supp} x_{k}^{R_{2}} \neq \emptyset$, we have that $w\left(f_{\beta_{t}}\right) \neq m_{2 j_{k}}$. Hence, apply Proposition 6.7 to obtain

$$
f\left(\sum_{k \in E^{c}} b_{k} x_{k}^{R_{2}}\right) \leq \frac{40 C}{\min _{\beta_{t} \in R^{2}} w\left(f_{\beta_{t}}\right)} \leq \frac{C}{m_{2 j+1}^{2}} .
$$


Finally we pass to estimate $f\left(\sum_{k=1}^{d} b_{k} x_{k}^{R_{1}}\right)$. For each $\beta_{t} \in R^{1}$ we denote by $S_{\beta_{t}}$ its immediate successors in $\mathcal{A}$. By the tree-like property of special sequences and the fact that $\left(x_{k}, \phi_{k}\right)_{k=1}^{d}$ is a 0 -dependent sequence, we can see that there exists at most one $\gamma_{\beta} \in S_{\beta}$ and at most one $k_{0} \in\{1, \ldots, d\}$ such that $\operatorname{supp} f_{\gamma_{\beta}} \cap \operatorname{supp} x_{k_{0}} \neq \emptyset$ with $w\left(f_{\gamma_{\beta}}\right)=m_{2 j_{k_{0}}}$. This observation yields that the family $\left\{f_{\gamma_{\beta}}: \beta \in R^{1}\right\}$ is $S_{n_{2 j+1}-1}$-allowable and that the same holds for $\left\{\operatorname{maxsupp} x_{k}^{R_{1}}: \exists \beta \in R^{1}, \gamma \in\right.$ $S_{\beta}$ with $\left.w\left(f_{\gamma}\right)=m_{2 j_{k}}\right\}$. Setting $E_{1}=\left\{k \leq d: \exists \beta \in R^{1}, \gamma \in S_{\beta}\right.$ with $w\left(f_{\gamma}\right)=$ $\left.m_{2 j_{k}}\right\}$ as a direct application of the upper $\ell_{2}$ estimate yields

$$
f\left(\sum_{k \in E_{1}} b_{k} x_{k}^{R_{1}}\right) \leq C\left(\sum_{k \in E_{1}} b_{k}^{2}\right)^{1 / 2} \leq \frac{C}{m_{2 j+1}^{2}} .
$$

For $k \notin E_{1}$

$$
f\left(\sum_{k \notin E_{1}} b_{k} x_{k}^{R_{1}}\right)=\sum_{\beta \in R^{1}} \frac{1}{m_{2 j+1}} \sum_{\gamma \in S_{\beta}} \frac{\lambda(\gamma)}{m(\gamma)} f_{\gamma}\left(\sum_{k \notin E_{1}} b_{k} x_{k}^{R_{1}}\right) .
$$

We may observe now that by the definition of the special sequences for each $\gamma \in \bigcup_{\beta \in R^{1}} S_{\beta}$, if there exists $k \notin E_{1}$ such that $\operatorname{supp} f_{\gamma} \cap \operatorname{supp} x_{k}^{R_{1}} \neq \emptyset$, then $w\left(f_{\gamma}\right)>m_{2 j+1}$ and $w\left(f_{\gamma}\right) \neq m_{2 j_{k}}$. Thus, applying Proposition 6.7 we obtain

$$
f\left(\sum_{k \notin E_{1}} b_{k} x_{k}^{R_{1}}\right) \leq \frac{40 C}{\min _{\gamma \in \cup_{\beta} S_{b}} w\left(f_{\gamma}\right)} \leq \frac{C}{m_{2 j+1}^{2}} .
$$

Proposition 6.10. Let $j \in \mathbb{N}, C>0$, and $\left(x_{k}\right)_{k=1}^{d}$ be $a(0, C, 2 j+1)$ dependent sequence and let $\sum_{k=1}^{d} b_{k} x_{k}$ be $a\left(1 / m_{2 j+2}^{2}, n_{2 j+1}\right)$-scc. Then

$$
\left\|\sum_{k=1}^{d} b_{k} x_{k}\right\| \leq \frac{C}{m_{2 j+1}^{2}} .
$$

Proof. Fix a $\mathcal{S}_{n_{2 j+1}} \sigma$-special sequence $\left(\phi_{k}\right)_{k=1}^{d}$ such that $m_{2 j_{k}}=w\left(\phi_{k}\right)$ for $k=$ $1, \ldots, d$ and let $f \in D_{w h}$. Let $\left(E_{k}, 2_{j_{k}}\right)_{k=1}^{d}$ be the $\mathcal{S}_{n_{2 j+1}} \sigma$-special sequence corresponding to $\left(\phi_{k}\right)_{k=1}^{d}$. If $w(f)<m_{2 j+1}$, Proposition 6.1 yields the result. Assuming that $w(f) \geq m_{2 j+1}$ we distinguish the following cases:

Case 1. $w(f) \geq m_{2 j+2}$. Assume that there exists a $k_{0} \in\{1, \ldots, d\}$ such that $w(f)=m_{2 j_{0}}$. Then

$$
f\left(\sum_{k=1}^{d} b_{k} x_{k}\right)=f\left(b_{k_{0}} x_{k_{0}}\right)+f\left(\sum_{k \neq k_{0}} b_{k} x_{k}\right) .
$$

By Proposition 6.7 $f\left(\sum_{k \neq k_{0}} b_{k} x_{k}\right) \leq \frac{40 C}{w(f)}$. Additionally, $f\left(b_{k_{0}} x_{k_{0}}\right)$ $\leq \| x_{k_{0}}|| b_{k_{0}} \mid \leq \frac{C}{m_{2 j+2}^{2}}$. Hence,

$$
f\left(\sum_{k=1}^{d} b_{k} x_{k}\right) \leq \frac{C}{m_{2 j+1}^{2}} .
$$

Case 2. $w(f)=m_{2 j+1}$. Then $f=\frac{1}{m_{2 j+1}} \sum_{\ell=1}^{r} \lambda_{\ell} f_{\ell}$ with $\left(f_{\ell}\right)_{\ell=1}^{r}$ being an $S_{n_{2 j+1}}$ $\sigma$-dependent sequence. Let $\left(F_{\ell}, 2_{j_{\ell}}\right)_{\ell=1}^{r}$ be the $\mathcal{S}_{n_{2 j+1}} \sigma$-special sequence corresponding to $\left(f_{\ell}\right)_{\ell=1}^{r}$. If there exists $k \in\{1, \ldots, d\}$ such that $\operatorname{supp} f \cap \operatorname{supp} x_{k} \neq \emptyset$, then by the tree-like property of special sequences there exists $\ell_{0} \in\{1, \ldots, r\}$ such that 
$F_{\ell}=E_{\ell}$ for all $\ell<\ell_{0}, w\left(f_{\ell_{0}}\right)=w\left(\phi_{\ell_{0}}\right)=m_{2 j_{\ell_{0}}}$ and $w\left(f_{\ell}\right) \neq m_{2 j_{k}}$ for all $\ell>\ell_{0}$ and

$k>\ell_{0}$. Since $\left(x_{k}\right)_{k=1}^{d}$ is 0 -dependent we have $\left(\bigcup_{\ell=1}^{\ell_{0}-1} \operatorname{supp} f_{\ell}\right) \cap\left(\bigcup_{k=1}^{d} \operatorname{supp} x_{k}\right)=\emptyset$. In this context we write

$$
\begin{aligned}
f\left(\sum_{k=1}^{d} b_{k} x_{k}\right)= & \frac{1}{m_{2 j+1}} \lambda_{\ell_{0}} f_{\ell_{0}}\left(\sum_{k=1}^{d} b_{k} x_{k}\right)+\frac{1}{m_{2 j+1}} \sum_{\ell>\ell_{0}} \lambda_{\ell} f_{\ell}\left(\sum_{k=1}^{d} b_{k} x_{k}\right) \\
= & \frac{1}{m_{2 j+1}} \lambda_{\ell_{0}} f_{\ell_{0}}\left(b_{\ell_{0}} x_{\ell_{0}}\right)+\frac{1}{m_{2 j+1}} \lambda_{\ell_{0}} f_{\ell_{0}}\left(\sum_{k \neq \ell_{0}} b_{k} x_{k}\right) \\
& +\frac{1}{m_{2 j+1}} \sum_{\ell>\ell_{0}} \lambda_{\ell} f_{\ell}\left(\sum_{k=1}^{d} b_{k} x_{k}\right) .
\end{aligned}
$$

For the first term, since $\sum_{k=1}^{d} b_{k} x_{k}$ is a $\left(\frac{1}{m_{2 j+2}^{2}}, n_{2 j+1}\right)$-scc we have, $\left|b_{\ell_{0}}\right|<\frac{1}{m_{2 j+2}^{2}}$. Therefore, $\frac{1}{m_{2 j+1}} \lambda_{\ell_{0}} f_{\ell_{0}}\left(b_{\ell_{0}} x_{\ell_{0}}\right) \leq \frac{C}{m_{2 j+2}^{2}}$. For the last two terms we apply Proposition 6.7 to see that

$$
\frac{1}{m_{2 j+1}} \lambda_{\ell_{0}} f_{\ell_{0}}\left(\sum_{k \neq \ell_{0}} b_{k} x_{k}\right) \leq \frac{40 C}{w\left(f_{\ell_{0}}\right)} \leq \frac{C}{m_{2 j+1}^{2}}
$$

and

$$
\frac{1}{m_{2 j+1}} \sum_{\ell>\ell_{0}} \lambda_{\ell} f_{\ell}\left(\sum_{k=1}^{d} b_{k} x_{k}\right) \leq \frac{40 C}{\min _{\ell>\ell_{0}} w\left(f_{\ell}\right)} \leq \frac{C}{m_{2 j+1}^{2}} .
$$

\section{THE SPACE OF BOUNDED LINEAR OPERATORS}

In this section we investigate the behavior of the operators in $\mathcal{L}(Y)$ where $Y$ is a block subspace of $\mathfrak{X}_{w h}$. In particular, we show that every $T \in \mathcal{L}(Y)$ takes the form $T=D+S$, where $D$ is diagonal and $S$ strictly singular. For that purpose we start by fixing $Y$ to be a subspace of $\mathfrak{X}_{w h}$ generated by a normalized block sequence $\left(y_{n}\right)_{n}$. We start with the following easy remark.

Remark 7.1. Let $\left(x_{n}\right)_{n}$ be a $\left(C, 2 j_{k}\right)_{k}$-RIS and $\left(B_{n}\right)_{n}$ be finite subsets of $\mathbb{N}$ such that $B_{n} \subset \operatorname{supp} x_{n}$ for all $n \in \mathbb{N}$. Then, the sequence $B_{n} x_{n}$ is a $\left(C, 2 j_{k}\right)_{k}$-RIS.

Lemma 7.2. Let $\left(x_{n}\right)_{n}$ be RIS in $Y$ and $T: Y \rightarrow Y$ be a bounded linear operator. Then for every $n$ and for every partition of $\operatorname{supp} x_{n}$ into sets $C_{n}, B_{n}$, $\lim _{n} C_{n} T B_{n}\left(x_{n}\right)=0$.

Proof. Suppose, towards a contradiction, that the conclusion fails. Then, by passing to a subsequence if necessary, we assume that there exists $\epsilon>0$ such that $\left\|C_{n} T B_{n}\right\|>\epsilon$ for every $n \in \mathbb{N}$. By Remark $7.1\left(B_{n} x_{n}\right)$ is a $\left(C,\left(2 i_{n}\right)\right)$-RIS. For each $n \in \mathbb{N}$ let $f_{n} \in D_{w h}$ such that $f_{n}\left(C_{n} T B_{n} x_{n}\right)>\varepsilon$ and $\operatorname{supp} f_{n} \subset C_{n}$. Choose $j \in \mathbb{N}$ such that $\frac{1}{m_{2 j+1}}<\frac{\varepsilon}{\|T\| C}$. Our goal is to construct sequences $\left(z_{k}\right)_{k=1}^{\infty},\left(g_{k}\right)_{k=1}^{\infty}$ and $\left(E_{i}, 2 j_{i}\right)_{i=1}^{\infty}$ such that:

(1) $\left(z_{k}\right)_{k=1}^{\infty}$ is a $(0, C, 2 j+1)$ dependent sequence with respect to $\left(E_{i}, 2 j_{i}\right)_{i=1}^{\infty}$;

(2) $3 / \operatorname{maxsupp} z_{1}<1 / m_{2 j+2}^{2}$;

(3) $\left(g_{k}\right)_{k=1}^{\infty} \subset D_{w h}$ is $\sigma$-special with respect to $\left(E_{i}, 2 j_{i}\right)_{i=1}^{\infty}$;

(4) $g_{k}\left(T z_{k}\right) \geq \varepsilon$ for all $k \in \mathbb{N}$. 
Assuming we can construct these sequences we arrive at a contraction in the following way: find $d \in \mathbb{N}$ such that $\left(\min E_{i}\right)_{i=1}^{d}$ is a maximal element of $\mathcal{S}_{n_{2 j+1}}$. Using Remark 3.11 there is a sequence $\left(b_{k}\right)_{k=1}^{d}$ such that $\sum_{k=1}^{d} b_{k} z_{k}$ is a $\left(1 / m_{2 j+2}^{2}, n_{2 j+1}\right)$ scc. Indeed, this is possible since minsupp $E_{i} \leq \operatorname{maxsupp} z_{i}$ for all $i \in \mathbb{N}$. Using the conditions on the sequences and Propostion 6.10 the contradiction to our choice of $j$ is as follows:

$$
\frac{\varepsilon}{m_{2 j+1}}<\frac{1}{m_{2 j+1}} \sum_{k=1}^{d} b_{k} g_{k}\left(\sum_{k=1}^{d} b_{k} T z_{k}\right) \leq\left\|T\left(\sum_{k=1}^{d} b_{k} z_{k}\right)\right\| \leq \frac{C\|T\|}{m_{2 j+1}^{2}} .
$$

Let us now construct the desired sequences. Let $j+1<j_{1} \in N_{1}$. Using Remark 3.11 find $F_{1} \subset \mathbb{N}$ and $\left(a_{1, n}\right)_{n \in F_{1}}$ such that $\sum_{n \in F_{1}} a_{1, n} B_{n} x_{n}$ is a $\left(1 / m_{2 j_{1}}^{3}, n_{2 j_{1}}\right)$-scc and $3 / \operatorname{maxsupp} x_{\max F_{1}}<1 / m_{2 j+2}^{2}$. Set

$$
z_{1}=m_{2 j_{1}} \sum_{n \in F_{1}} a_{1, n} B_{n} x_{n} \text { and } g_{1}=\frac{1}{m_{2 j_{1}}} \sum_{n \in F_{1}} a_{1, n} f_{n} .
$$

Notice that $\operatorname{supp} g_{1} \cap \operatorname{supp} z_{1}=\emptyset$ and $g_{1}\left(T z_{1}\right)>\varepsilon$. Let $E_{1}=\operatorname{supp} g_{1} \cup\left\{\operatorname{maxsupp} z_{1}+\right.$ $1\}$ and $j_{2}=\sigma\left(E_{1}, 2 j_{1}\right)$. Find $F_{2}>\max F_{1}+1$ and $\left(a_{2, n}\right)_{n \in F_{2}}$ such that $\sum_{n \in F_{2}} a_{2, n} B_{n} x_{n}$ is a $\left(1 / m_{2 j_{2}}^{3}, n_{2 j_{2}}\right)$-scc. Set

$$
z_{2}=m_{2 j_{2}} \sum_{n \in F_{2}} a_{2, n} B_{n} x_{n} \text { and } g_{2}=\frac{1}{m_{2 j_{2}}} \sum_{n \in F_{2}} a_{2, n} f_{n} .
$$

Notice that $\left(\operatorname{supp} g_{1} \cup \operatorname{supp} g_{2}\right) \cap\left(\operatorname{supp} z_{1} \cup \operatorname{supp} z_{2}\right)=\emptyset$. Let $E_{2}=\operatorname{supp} g_{2} \cup$ $\left\{\right.$ maxsupp $\left.z_{2}+1\right\}$. By continuing in this manner we construct the desired sequences. Notice that since maxsupp $z_{k}<\operatorname{maxsupp} E_{k}$ and $\left(\bigcup_{k} E_{k}\right) \cap\left(\bigcup_{k} \operatorname{supp} x_{k}\right)=\emptyset$, we have that $\left(z_{k}\right)$ is a $(0, C, 2 j+1)$ dependent sequence with respect to $\left(E_{i}, 2 j_{i}\right)_{i=1}^{\infty}$.

The following can be readily verified.

Remark 7.3. Let $\left(x_{n}\right)_{n}$ be RIS in $Y$ and $T: Y \rightarrow Y$ be a bounded linear operator. Set $s_{n}=\sup \left\|C_{n} T B_{n} x_{n}\right\|$, for $n \in \mathbb{N}$, where the supremum is taken over all partitions $\left(B_{n}, C_{n}\right)$ of $\operatorname{supp} x_{n}$. Then $\lim _{n} s_{n}=0$.

The next step is to show that every diagonal free operator $T: Y \rightarrow Y$ has the property that $T x_{n} \rightarrow 0$ for every RIS sequence $x_{n}$ in $Y$. By diagonal free we mean that $y_{n}^{*}\left(T y_{n}\right)=0$ for every $n \in \mathbb{N}$.

To prove this result we will need a preparatory lemma that uses a simple counting argument and is due to W.T. Gowers and B. Maurey [17]. Its present form is taken from Proposition 9.3 in [7. Before we state the lemma let us fix some notation.

Let $T: Y \rightarrow Y$ be a bounded linear operator and suppose that $\left(x_{n}\right)_{n}$ is a block sequence. For each $n \in N$ we define the following:

(1) $A_{n}=\operatorname{supp} x_{n}$.

(2) $P_{n}=\left\{(B, C): B \cup C=A_{n}, B \cap C=\emptyset, \# B=\frac{\# A_{n}}{2}\right\}$ if $\# A_{n}$ is even.

(3) $P_{n}=\left\{(B, C): B \cup C=A_{n}, B \cap C=\emptyset,|\# B-\# C|=1\right\}$ if $\# A_{n}$ is odd.

(4) We set $L_{n}$ to be the entire part of $\frac{\# A_{n}}{2}$.

Lemma 7.4. Let $T \in \mathcal{L}(Y)$ be diagonal free and $\left(x_{n}\right)_{n}$ be a block sequence in $Y$. Then we have the following:

i. $A_{n} T x_{n}=\frac{2 L_{n}\left(2 L_{n}-1\right)}{L_{n}^{2}} \frac{1}{\# P_{n}} \sum_{(B, C) \in P_{n}} B T C x_{n}$ if \# $A_{n}$ is even.

ii. $A_{n} T x_{n}=\frac{2 L_{n}\left(2 L_{n}+1\right)\left(\left(L_{n}+1\right)^{2}+1\right)}{\left(L_{n}^{2}+1\right)\left(L_{n}+1\right)^{2}} \frac{1}{\# P_{n}} \sum_{(B, C) \in P_{n}} B T C x_{n}$ if \# $A_{n}$ is odd. 
Proof. We fix $n \in \mathbb{N}$. We give the proof only in the case where $\# A_{n}$ is even, as the other case is similar. We can write $x_{n}$ as $x_{n}=\sum_{k=1}^{d} a_{k} y_{k}$. Then $A_{n} T x_{n}=$ $\sum_{i \in A_{n}}\left(\sum_{k=1}^{d} a_{k} y_{i}^{*}\left(T y_{k}\right)\right) y_{i}$. As the operator is diagonal free we can rewrite the above sum as

$$
A_{n} T x_{n}=\sum_{i \in A_{n}} \sum_{k \neq i} a_{k} y_{i}^{*}\left(T y_{k}\right) y_{i} .
$$

We fix $i \in A_{n}$ and pass to show that

$$
\left(\sum_{k \neq i} a_{k} y_{i}^{*}\left(T y_{k}\right)\right)=\frac{2 L_{n}\left(2 L_{n}-1\right)}{L_{n}^{2}} \frac{1}{\# P_{n}} \sum_{(B, C) \in P_{n}} y_{i}^{*}\left(B T C x_{n}\right) .
$$

For a fixed pair $(B, C) \in P_{n}$ we have that $y_{i}^{*}\left(B T C x_{n}\right)=\sum_{k \in C} a_{k} y_{i}^{*} T\left(y_{k}\right)$, which is non-zero only if $i \notin C$, as $B, C$ form a partition of $A_{n}$ and $T$ is diagonal free. This indicates that for each $k \neq i$ the term $a_{k} y_{i}^{*}\left(T y_{k}\right)$ appears in the sum $\sum_{(B, C) \in P_{n}} y_{i}^{*}\left(B T C x_{n}\right)$ as many times as is the cardinality of the set $R_{n}=\{C \subset$ $A_{n}: i \notin C, k \in C$ and $\left.\# C=\frac{\# A_{n}}{2}=L_{n}\right\}$. We can easily see that

$$
\# R_{n}=\frac{\left(2 L_{n}-2\right) !}{\left(\left(L_{n}-2\right) !\right)^{2}}=\frac{2 L_{n}\left(2 L_{n}-1\right)}{L_{n}^{2}} \cdot \frac{1}{\# P_{n}} .
$$

This completes the proof.

We can now show the following.

Proposition 7.5. Let $T: Y \rightarrow Y$ be a diagonal free bounded linear operator. Then for every $\left(C, 2 j_{k}\right)_{k}-R I S\left(x_{n}\right)_{n}$ in $Y, T\left(x_{n}\right) \rightarrow 0$. In particular, $T$ is strictly singular.

Proof. Assume that the conclusion fails and by passing to a subsequence if necessary we suppose that $\left\|T x_{n}\right\|>\epsilon$ for all $n \in \mathbb{N}$.

First we observe that $\lim _{n} A_{n} T\left(x_{n}\right)=0$. Indeed, by Lemma 7.4 for each $n \in \mathbb{N}$ we can write $A_{n} T x_{n}=\lambda_{n} \frac{1}{\# P_{n}} \sum_{(B, C) \in P_{n}} B T C x_{n}$, where $1 \leq \lambda_{n} \leq 4$. Therefore using Remark 7.3 we obtain $\lim _{n} A_{n} T\left(x_{n}\right)=0$. Granting this and using a sliding hump argument we may assume the following two properties concerning the sequences $\left(x_{n}\right)_{n}$ and $\left(T x_{n}\right)$ :

(1) The sequence $\left(T x_{n}\right)_{n}$ is a block sequence.

(2) $\operatorname{supp} x_{n} \cap \operatorname{supp} T x_{n}=\emptyset$, for all $n \in \mathbb{N}$.

We choose a $j \in \mathbb{N}$ such that $\frac{1}{m_{2 j+1}}<\frac{\epsilon}{C\|T\|}$. Following the same lines as in the proof of Lemma 7.2 we inductively construct sequences $\left(z_{k}\right)_{k=1}^{\infty},\left(g_{k}\right)_{k=1}^{\infty}$ and $\left(E_{i}, 2 j_{i}\right)_{i=1}^{\infty}$ such that:

(1) $\left(z_{k}\right)_{k=1}^{\infty}$ is a $(0, C, 2 j+1)$ dependent sequence with respect to $\left(E_{i}, 2 j_{i}\right)_{i=1}^{\infty}$;

(2) $3 / \operatorname{maxsupp} z_{1}<1 / m_{2 j+2}^{2}$;

(3) $\left(g_{k}\right)_{k=1}^{\infty} \subset D_{w h}$ is $\sigma$-special with respect to $\left(E_{i}, 2 j_{i}\right)_{i=1}^{\infty}$;

(4) $g_{k}\left(T z_{k}\right) \geq \varepsilon$ for all $k \in \mathbb{N}$.

Granting this, find $d \in \mathbb{N}$ such that $\left(\min E_{i}\right)_{i=1}^{d}$ is a maximal element of $\mathcal{S}_{n_{2 j+1}}$. Using Remark 3.11 there is a sequence $\left(b_{k}\right)_{k=1}^{d}$ such that $\sum_{k=1}^{d} b_{k} z_{k}$ is a $\left(1 / m_{2 j+2}^{2}\right.$, $n_{2 j+1}$ )-scc. Using Proposition 6.10 the contradiction to our choice of $j$ is as follows:

$$
\frac{\varepsilon}{m_{2 j+1}}<\frac{1}{m_{2 j+1}} \sum_{k=1}^{d} b_{k} g_{k}\left(\sum_{k=1}^{d} b_{k} T z_{k}\right) \leq\left\|T\left(\sum_{k=1}^{d} b_{k} z_{k}\right)\right\| \leq \frac{C\|T\|}{m_{2 j+1}^{2}} .
$$


All the above yield the following:

Proposition 7.6. Let $Y$ be a block subspace of $\mathfrak{X}_{w h}$ and $T: Y \rightarrow Y$ a bounded linear operator. Then $T$ has the form $T=D+S$, where $D: Y \rightarrow Y$ is a diagonal operator and $S: Y \rightarrow Y$ is strictly singular.

Proof. We set $D\left(y_{n}\right)=y_{n}^{*}\left(T y_{n}\right) y_{n}$. Then clearly $D$ is diagonal and bounded. By the previous proposition we have that $T-D$ is a strictly singular operator.

As a consequence we obtain the following:

Theorem 7.7. Let $Y$ be a block subspace of $\mathfrak{X}_{w h}$. Then $Y$ is not isomorphic to any of its proper subspaces.

For a proof of the above we refer the interested reader to Corollary 30 in [17.

Similar arguments as the ones used in the preceding theorem and Proposition 7.6 can be applied to a more general setting. Namely, if $Y$ is a subspace generated by a sequence $\left(y_{n}\right)_{n=1}^{\infty}$ of disjointly supported vectors and $T \in \mathcal{L}(Y)$, then $T$ takes the form $T=D+S$, where $D$ is diagonal and $S$ strictly singular.

Remark 7.8. Let $Y$ be a subspace of $\mathfrak{X}_{w h}$ generated by a sequence $\left(y_{n}\right)_{n=1}^{\infty}$ with pairwise disjoint supports and let $T \in \mathcal{L}(Y)$. Then there exists a diagonal operator $D^{\prime}: \mathfrak{X}_{w h} \rightarrow \mathfrak{X}_{w h}$ and a strictly singular operator $S \in \mathcal{L}(Y)$ such that $T=\left.D^{\prime}\right|_{Y}+S$.

Proof. By Proposition 7.6 there exist a diagonal operator $D \in \mathcal{L}(Y)$ and a strictly singular $S \in \mathcal{L}(Y)$ such that $T=D+S$. For each $n \in \mathbb{N}$ there exists a $\lambda_{n} \in \mathbb{R}$ such that $T\left(y_{n}\right)=\lambda_{n} y_{n}$. Let $i \in \mathbb{N}$. Set $D^{\prime} e_{i}=\lambda_{n} e_{i}$ if $i \in \operatorname{supp} y_{n}$ and $D^{\prime} e_{i}=0$ otherwise. It can be readily seen that $D_{Y}$ is a diagonal operator in $\mathcal{L}\left(\mathfrak{X}_{w h}\right)$ and that the restriction of $D^{\prime}$ on $Y$ coincides with $D$.

This remark gives rise to the following problem.

Problem 3. Let $Z$ be an arbitrary infinite dimensional closed subspace of $\mathfrak{X}_{w h}$. Let $T: Z \rightarrow Z$ be a bounded linear operator. Do there exist $D: \mathfrak{X}_{w h} \rightarrow \mathfrak{X}_{w h}$ diagonal and $S$ strictly singular such that $T=\left.D\right|_{Z}+S$ ? Moreover, does there exist an infinite dimensional closed subspace $Z$ of $\mathfrak{X}_{w h}$ which is isomorphic to one of its proper subspaces?

The arguments in the proof of Proposition 7.6 yield the following.

Proposition 7.9. Let $Y, Z$ be two disjointly supported block subspaces of $\mathfrak{X}_{w h}$. Then every bounded linear operator $T: Y \rightarrow Z$ is strictly singular.

We recall that a Banach space $X$ is quasi-minimal if every two infinite dimensional closed subspaces of $X$ are not totally incomparable.

Corollary 7.10. The space $\mathfrak{X}_{w h}$ does not contain a quasi-minimal subspace.

Proof. Suppose $Z$ is a quasi-minimal subspace of $\mathfrak{X}_{w h}$. Let $Y$ be a subspace of $Z$ isomorphic to a subspace of $\mathfrak{X}_{w h}$ generated by a block sequence $\left(y_{n}\right)_{n \in \mathbb{N}}$. Proposition 7.9 yields that the spaces generated by $\left(y_{2 n}\right)_{n \in \mathbb{N}}$ and $\left(y_{2 n+1}\right)_{n \in \mathbb{N}}$ are totally incomparable. This contradicts the assumption that $Y$ is quasi-minimal, and the proof is complete.

As was mentioned in the introduction, using the same method one can construct a space $\mathfrak{X}_{w h}^{C}$ over the field of complex numbers that shares the same properties as 
$\mathfrak{X}_{w h}$. At this point we consider some of the spectral properties of a $T \in \mathcal{L}\left(\mathfrak{X}_{w h}\right)$. For the rest of this section we abuse notation and denote by $\mathfrak{X}_{w h}$ both the real and the complex Banach space discussed above. For every bounded linear operator $T$ that is considered we let $\sigma(T)$ be its spectrum and $\sigma_{p}(T)$ its point spectrum. We start with the following result.

Lemma 7.11. Let $D \in \mathcal{L}\left(\mathfrak{X}_{w h}\right)$ be a diagonal operator with $D e_{n}=\lambda_{n} e_{n}$, for $n \in \mathbb{N}$. Then $\sigma(D)=\overline{\left\{\lambda_{n}\right\}_{n=1}^{\infty}}$.

Proof. Let $\lambda \in \overline{\left\{\lambda_{n}\right\}_{n=1}^{\infty}}$. Choose a subsequence $\left(\lambda_{k_{n}}\right)_{n=1}^{\infty}$ such that $\lambda_{k_{n}} \rightarrow \lambda$. Observe that $D e_{k_{n}}-\lambda e_{k_{n}} \rightarrow 0$ and thus $\lambda \in \sigma(D)$. Now, suppose that $\lambda \notin \overline{\left\{\lambda_{n}\right\}_{n=1}^{\infty}}$. Then there exists $\epsilon>0$ with $\left|\lambda-\lambda_{n}\right|>\epsilon$. As the basis of $\mathfrak{X}_{w h}$ is unconditional, this yields that $D-\lambda I$ is invertible, and the proof is complete.

The following result correlates the spectrum of an arbitrary $T \in \mathcal{L}\left(\mathfrak{X}_{w h}\right)$ with that of its diagonal part.

Proposition 7.12. Let $T \in \mathcal{L}\left(\mathfrak{X}_{w h}\right)$ with $T=D+S$, where $D, S \in \mathcal{L}\left(\mathfrak{X}_{w h}\right)$ and $D$ is diagonal and $S$ strictly singular. Then the following hold:

(1) $\sigma(T) \backslash\left(\sigma_{p}(T) \cup\{0\}\right) \subset \sigma(D)$.

(2) If $\lambda_{n}$ is an eigenvalue of $D$ with infinite dimensional eigenspace, then $\lambda_{n} \in$ $\sigma(T)$.

(3) $\sigma(D) \backslash\left(\sigma_{p}(D) \cup\{0\}\right) \subset \sigma(T)$.

Proof. (1) Let $\lambda \in \sigma(T) \backslash\left(\sigma_{p}(T) \cup\{0\}\right)$ and suppose towards a contradiction that $\lambda \notin$ $\sigma(D)$. Then the operator $D-\lambda I$ is invertible and therefore a Fredholm operator of index 0. Standard Fredholm theory (see for example Proposition 2.c.10 in [22]) that $D+S-\lambda I$ is also Fredholm of index zero. By our assumptions, $T-\lambda I=D+S-\lambda I$ is not invertible, and as it is Fredholm, $\lambda \in \sigma_{p}(T)$, which is a contradiction, and the proof is complete.

(2) By our assumptions there exists an infinite dimensional subspace $Y$ of $\mathfrak{X}_{w h}$ such that $\left.\left(D-\lambda_{n} I\right)\right|_{Y}=0$. Therefore, $\left.\left(T-\lambda_{n} I\right)\right|_{Y}=\left.S\right|_{Y}$, which immediately yields that $T-\lambda_{n} I$ is not invertible.

(3) Let $\lambda \in \overline{\left\{\lambda_{n}\right\}_{n=1}^{\infty}}$ with $\lambda \notin\left\{\lambda_{n}: n \in \mathbb{N}\right\} \cup\{0\}$. Choose a subsequence $\left(\lambda_{k_{n}}\right)_{n=1}^{\infty}$ such that $\lambda_{k_{n}} \rightarrow \lambda$. Since $D e_{k_{n}}-\lambda e_{k_{n}} \rightarrow 0$ and $S$ is strictly singular, we can find a subspace $Y$ of $\left\langle e_{k_{n}}: n \in \mathbb{N}>\right.$ such that the operators $D-\lambda I$ and $S$ are both compact on $Y$. Hence, $T-\lambda I$ is not invertible.

\section{The proof of Proposition 6.7}

The aim of this section is primarily to prove Proposition 6.7 which was stated in Section 6. We start with the following.

Proposition 8.1. Let $\left(x_{k}\right)_{k=1}^{d}$ be a $\left(C, 2 j_{k}\right)$ an exact sequence. Let $\left(f_{\ell}\right)_{\ell=1}^{r} \subset$ $D_{w h}$ be $\mathcal{S}_{n_{q}}$-allowable with $q<2 j_{1}$. Assume further that for each $k \in\{1, \ldots, d\}$, $\max \left\{\omega\left(f_{\ell}\right): \operatorname{supp} x_{k} \cap \operatorname{supp} f_{\ell} \neq \emptyset\right\}<m_{2 j_{k}}$. Then for all $\left(\lambda_{\ell}\right)_{\ell=1}^{r},\left(b_{k}\right)_{k=1}^{d} \in B a\left(\ell_{2}\right)$,

$$
\sum_{\ell=1}^{r} \lambda_{\ell} f_{\ell}\left(\sum_{k=1}^{d} b_{k} x_{k}\right) \leq \frac{16 C}{\min _{1 \leq \ell \leq r} \omega\left(f_{\ell}\right)} .
$$


For the proof of Proposition 8.1 we shall follow a similar strategy to that of Proposition 6.1. Namely, we shall first consider a partition of the vectors $\left(x_{k}\right)_{k=1}^{d}$ and then proceed with the evaluation of $\sum_{\ell=1}^{r} \lambda_{\ell} f_{\ell}$ on each part separately.

For each $k \in\{1, \ldots, d\}$, let $x_{k}=m_{2 j_{k}} \sum_{i=1}^{p_{k}} b_{k, i} x_{k, i}$, where $\left(\left(x_{k, i}\right)_{i=1}^{p_{k}}\right)_{k=1}^{d}$ is a $\left(C,\left(2 j_{k, i}\right)\right)$ RIS sequence in the lexicographical ordering. Recall that $j_{k}<j_{k, i}<$ $j_{k+1}$ for all $k \in\{1, \ldots, d\}$ and $i \in\left\{1, \ldots, p_{k}\right\}$. We will partition the support of each $x_{k, i}$. For each $k \in\{1, \ldots, d\}$ and $i \in\left\{1, \ldots, p_{k}\right\}$ let

$$
G_{k, i}=\left\{t \in \mathcal{A}: t \text { is maximal } \operatorname{supp} f_{t} \cap \operatorname{supp} x_{k, i} \neq \emptyset, \frac{1}{m(t)} \leq \frac{1}{m_{2 j_{k, i+1}}}\right\} .
$$

Let $P_{k, i}$ denote the maximal $t \in \mathcal{A}$ such that supp $f_{t} \cap \operatorname{supp} x_{k, i} \neq \emptyset$ and $t \notin G_{k, i}$.

For $t \in P_{k, i}$, let $\beta_{t}=t$ if $w\left(f_{\gamma}\right)<m_{2 j_{k}}$ for all $\gamma \prec t$ or $\beta_{t}=\min \left\{\gamma \prec t: w\left(f_{\gamma}\right) \geq\right.$ $\left.m_{2 j_{k}}\right\}$ otherwise.

We set $S_{k, i}=\left\{\beta_{t}: t \in P_{k, i}, \frac{1}{m\left(\beta_{t}\right)} \leq \frac{1}{m_{2 j_{k}}^{3}}\right\}, R_{k, i}=\left\{\beta_{t}: t \in P_{k, i}, \frac{1}{m\left(\beta_{t}\right)}>\frac{1}{m_{2 j_{k}}^{3}}\right\}$.

We split $R_{k, i}$ into $R_{k, i}^{1}=\left\{\beta_{t} \in R_{k, i}: w\left(f_{\beta_{t}}\right) \geq m_{2 j_{k, i}}\right\}, R_{k, i}^{2}=\left\{\beta_{t} \in R_{k, i}\right.$ : $\left.w\left(f_{\beta_{t}}\right)<m_{2 j_{k, i}}\right\}$.

Using the above sets we partition $x_{k, i}$ in the following way. Set $x_{k, i}^{G}=$ $x_{k, i}\left|\bigcup_{t \in G_{k, i}} \operatorname{supp} f_{t}, x_{k, i}^{S}=x_{k, i}\right|_{\bigcup_{\beta_{t} \in S_{k, i}}} \operatorname{supp} f_{\beta_{t}}, x_{k, i}^{R_{1}}=\left.x_{k, i}\right|_{\bigcup_{\beta_{t} \in R_{k, i}^{1}}} \operatorname{supp} f_{\beta_{t}}$ and $x_{k, i}^{R_{2}}=$ $x_{k, i} \mid \bigcup_{\beta_{t} \in R_{k, i}^{2}} \operatorname{supp} f_{\beta_{t}}$.

We also set $x_{k}^{I}=m_{2 j_{k}} \sum_{i=1}^{p_{k}} b_{k, i} x_{k, i}^{I}$, where $I=G, S, R^{1}, R^{2}$, and we denote by $G_{k}, S_{k}, R_{k}^{1}, R_{k}^{2}$ the union for all $i$ of the corresponding $G_{k, i}, S_{k, i}, R_{k, i}^{1}, R_{k, i}^{2}$.

We present the proof of Proposition 8.1

Proof. (1) We start by showing that $\sum_{\ell=1}^{r} \lambda_{\ell} f_{\ell}\left(\sum_{k=k_{0}}^{d} b_{k} x_{k}^{G}\right)<\frac{2}{m_{2 j_{1}}^{2}}$. We can readily see that $\left\|\left.f_{\ell}\right|_{\operatorname{supp} x_{k, i}^{G}}\right\|_{\infty} \leq \frac{1}{m_{2 j_{k, i+1}}}$ for all $\ell=1, \ldots, r, k=1, \ldots, d$ and $i=1, \ldots, p_{k}$. Thus applying the RIS estimate (RISE) on $x_{k}^{G}$ we can see that $\sum_{\ell=1}^{r} \lambda_{\ell} f_{\ell}\left(x_{k}^{G}\right) \leq \frac{1}{m_{2 j_{k}}^{2}}$. Summing up for all $k$ yields the result.

(2) We pass to the following evaluation:

$$
\sum_{\ell=1}^{r} \lambda_{\ell} f_{\ell}\left(\sum_{k=1}^{d} b_{k} x_{k}^{S}\right)<\frac{10 C}{m_{2 j_{1}}}
$$

For each $\beta_{t} \in S_{k}$ set $a_{t}=\min \left\{a \prec \beta_{t}: \frac{1}{m(a)}<\frac{1}{m_{2 j_{k}}^{2}}\right\}$. Observe that $\left\{a_{t}: \beta_{t} \in S_{k}\right\}$ is a maximal antichain for the tree representation of the functional $\left.\sum_{\ell=1}^{r} \lambda_{\ell} f_{\ell}\right|_{\operatorname{supp} x_{k}^{G}}$. We can also see that $\frac{1}{m\left(a_{t}\right)} \geq \frac{1}{m_{2 j_{k}}^{3}}$. Hence by Lemma 4.9 the family $\left\{f_{a_{t}}: \beta_{t} \in S_{k}\right\}$ is $S_{n_{2 j k}-1 \text {-allowable. Then }}$

$$
\sum_{\ell=1}^{r} \lambda_{\ell} f_{\ell}\left(x_{k}^{S}\right) \leq \frac{1}{m_{2 j_{k}}} \sum_{\left\{a_{t}: \beta_{t} \in S_{k}\right\}} \frac{\lambda\left(a_{t}\right)}{m\left(a_{t}\right)} f_{a_{t}}\left(\sum_{i=1}^{p_{k}} b_{k, i} x_{k, i}^{S}\right) .
$$

A direct application of Lemma 6.5 and a summation over all $k$ yields the estimate.

(3) At this point we prove that

$$
\sum_{\ell=1}^{r} \lambda_{\ell} f_{\ell}\left(\sum_{k=1}^{d} b_{k} x_{k}^{R_{1}}\right)<\frac{2 C}{m_{2 j_{1}}} .
$$


Fix $k \in\{1, \ldots, d\}$. For $\beta \in R_{k}^{1}$ let $h_{\beta}=\left.f_{\beta}\right|_{\operatorname{supp} x_{k}^{R_{1}}}$. Observe that

$$
\sum_{\ell=1}^{r} \lambda_{\ell} f_{\ell}\left(x_{k}^{R_{1}}\right)=m_{2 j_{k}} \sum_{\beta \in R_{k}^{1}} \frac{\lambda(\beta)}{m(\beta)} h_{\beta}\left(\sum_{i=1}^{p_{k}} b_{k, i} x_{k, i}^{R_{1}}\right) .
$$

By Remark 4.8 and the fact that for $k \neq k^{\prime}$ it holds that $R_{k}^{1} \cap R_{k^{\prime}}^{1}=\emptyset$. The family $\left\{h_{\beta}: \beta \in R_{k}^{1}\right\}$ is $S_{n_{2 j_{k}}-1^{-a l l o w a b l e}}$ and $\left\{\operatorname{maxsupp} x_{k, i}^{R_{1}}: i \leq p_{k}, x_{k, i}^{R_{1}} \neq 0\right\} \in S_{n_{2 j_{k}}-1^{-}}$ allowable. Therefore, $\frac{1}{m_{2 j_{k}}} \sum_{b \in R_{k}^{1}} \frac{\lambda(\beta)}{m(\beta)} h_{\beta}=g_{k} \in D_{w h}$ and $\left(\sum_{\left\{i: x_{k, i}^{R_{1}} \neq 0\right\}} b_{k, i}^{2}\right)^{1 / 2}<$ $\frac{1}{m_{2 j_{k}}^{3}}$.

Thus applying the upper $\ell_{2}$ estimate,

$$
\sum_{\ell=1}^{r} \lambda_{\ell} f_{\ell}\left(x_{k}^{R_{1}}\right)=m_{2 j_{k}}^{2} g_{k}\left(\sum_{\substack{\left.R_{k, i}^{R_{1}} \neq 0\right\} \\ x_{k, i}}} b_{k, i} x_{k, i}^{R_{1}}\right) \leq \frac{C}{m_{2 j_{k}}} .
$$

A summation over all $k$ yields the estimate.

(4) The last estimate is

$$
\sum_{\ell=1}^{r} \lambda_{\ell} f_{\ell}\left(\sum_{k=1}^{d} b_{k} x_{k}^{R_{2}}\right)<\frac{12 C}{\min _{1 \leq \ell \leq r} \omega\left(f_{\ell}\right)}
$$

For $k \in\{1, \ldots, d\}$ and $\beta \in R_{k}^{2}$, let $h_{\beta}=\left.f_{\beta}\right|_{\operatorname{supp} x_{k}^{R_{2}}}$. It is clear that for $\beta \neq \beta^{\prime}$, $\operatorname{supp} f_{\beta} \cap \operatorname{supp} f_{\beta^{\prime}}=\emptyset$. For $\beta \in R_{k}^{2}$ not maximal let $S_{\beta}$ denote its immediate successors in $\mathcal{A}$. For $\gamma \in S_{\beta}$ set $h_{\gamma}=\left.f_{\gamma}\right|_{\operatorname{supp} x_{k}^{R_{2}}}$. For given $k \in\{1, \ldots, d\}$ and $i \in\left\{1, \ldots, p_{k}\right\}$ write $x_{k, i}^{R_{2}}=\sum_{j=1}^{p_{k, i}} b_{k, i, j} x_{k, i, j}^{R_{2}}$. We define the following sets:

$$
\begin{gathered}
\Phi^{C}=\left\{(k, i, j): \exists \beta \in R_{k}^{2} \text { not maximal, } \gamma \in S_{\beta} \text { with minsupp } h_{\gamma} \in \operatorname{ran} x_{k, i, j}^{R_{2}}\right\} \\
\cup\left\{(k, i, j): \exists \beta \in R_{k}^{2} \text { maximal s.t. supp } f_{\beta} \cap \operatorname{supp} x_{k, i, j}^{R} \neq \emptyset\right\}, \\
\Phi_{k, i}^{C}=\left\{j:(k, i, j) \in \Phi^{C}\right\}, \Phi=\left\{(k, i, j): k \leq d, i \leq p_{k}, j \leq p_{k, i}\right\} \backslash \Phi, \\
\Phi_{k, i}=\left\{1, \ldots, p_{k, i}\right\} \backslash \Phi_{k, i}^{C} .
\end{gathered}
$$

Since, $w\left(f_{\beta}\right)<m_{2 j_{k, i}}$, for all $\beta \in R_{k, i}^{2}$ it follows that the family $\left\{h_{\gamma}: \gamma \in S_{\beta}\right\}$ is an $S_{n_{\left(2 j_{k, i}-1\right)}}$-allowable family. In addition, the family $\left\{h_{\beta}: \beta \in R_{k}^{2}\right\}$ is $S_{n_{\left(2 j_{k}-1\right)}}{ }^{-}$ allowable, hence the family $\left\{h_{\gamma}: \gamma \in S_{\beta}, \beta \in R_{k}^{2}\right\} \cup\left\{h_{\beta}: \beta\right.$ is maximal and $\operatorname{supp} h_{\beta}$ $\left.\cap \operatorname{supp} x_{k}^{R_{2}} \neq \emptyset\right\}$ is $S_{\left(n_{2 j_{k}}-1\right)}$-allowable. Hence, applying Lemma 6.3 (SAE),

$$
\left(\sum_{\beta \in R_{k}^{2}, \gamma \in S_{\beta}} \frac{\lambda(\gamma)}{m(\gamma)} h_{\gamma}+\sum_{\substack{\beta \in \mathcal{R}_{k}^{2} \\ \beta \text { maximal }}} \frac{\lambda(\beta)}{m(\beta)} f_{\beta}\right)\left(\sum_{j \in \Phi_{k, i}^{C}} b_{k, i, j} x_{k, i, j}^{R_{2}}\right) \leq \frac{C}{m_{2 j_{k, i}}} .
$$

Summing up for all $i \in\left\{1, \ldots, p_{k}\right\}$ and for all $k \in\{1, \ldots, d\}$ yields the desired estimate.

The final estimate for this proposition concerns $x_{k, i}^{R_{2}}$ such that $j \in \Phi_{k, i}$. 
Before proceeding we need the following notion:

We call $\beta \in R^{2} s$-minimal if $\left|\left\{\beta^{\prime}: \beta^{\prime} \prec \beta, \beta^{\prime} \in R^{2}\right\}\right|=s$. For $s \geq 0$ let $L_{s}=\left\{\beta \in \mathcal{R}^{2}: \beta\right.$ is $s$-minimal $\}$.

Observe that for $s \geq 0$ and $\beta \neq \beta^{\prime}$ in $L_{s}$ the nodes $\beta, \beta^{\prime}$ are incomparable. Indeed, if we assume that $\beta \prec \beta^{\prime}$, then $\left|\left\{\gamma \in R^{2}: \gamma \prec \beta^{\prime}\right\}\right|=s \geq \mid\left\{\gamma \in R^{2}: \gamma \prec\right.$ $\beta\} \mid+1=s+1$, which is a contradiction showing that the nodes are incomparable.

Sublemma 8.2. For each $\gamma \in \bigcup S_{\beta}$ let, $\eta_{\gamma}=\lambda(\gamma) \prod_{\ell \prec \delta \prec \beta} \frac{1}{\omega\left(f_{\delta}\right)}$. Then $\sum_{\gamma \in \bigcup S_{\beta}} \eta_{\gamma}^{2}$ $\leq 2$.

Proof. By the definition of the set $R^{2}$ we deduce that for $\beta \in L_{s}$ with $s>0$, $\prod_{\ell \prec \delta \prec \beta} \frac{1}{\omega\left(f_{\delta}\right)}<\frac{1}{m_{2 j_{s}}}$. As we saw earlier, for each $s>0$ the nodes in $L_{s}$ are incomparable. Therefore,

$$
\begin{aligned}
\sum_{\gamma \in \cup S_{\beta}} \eta_{\gamma}^{2} & =\sum_{\ell=1}^{r} \lambda_{\ell}^{2} \sum_{\beta \in R^{2} \cap \mathcal{A}_{\ell}}\left(\prod_{\ell \prec \delta \prec \beta} \frac{\lambda_{\delta}}{\omega\left(f_{\delta}\right)}\right)^{2} \sum_{\gamma \in S_{\beta}} \lambda_{\gamma}^{2} \\
= & \sum_{s>0} \sum_{\ell=1}^{r} \lambda_{\ell}^{2} \sum_{\beta \in L_{s} \cap R^{2} \cap \mathcal{A}_{\ell}}\left(\prod_{\ell \prec \delta \prec \beta} \frac{\lambda_{\delta}}{\omega\left(f_{\delta}\right)}\right)^{2} \sum_{\gamma \in S_{\beta}} \lambda_{\gamma}^{2} \\
& \quad+\sum_{\ell=1}^{r} \lambda_{\ell}^{2} \sum_{\beta \in L_{0} \cap R^{2} \cap \mathcal{A}_{\ell}}\left(\prod_{\ell \prec \delta \prec \beta} \frac{\lambda_{\delta}}{\omega\left(f_{\delta}\right)}\right)^{2} \sum_{\gamma \in S_{\beta}} \lambda_{\gamma}^{2} \\
\leq & \left(\sum_{s>0} \frac{1}{m_{2 j_{s}}}\right)+1<2 .
\end{aligned}
$$

Now, let

$$
y=\left(\sum_{k=1}^{d} b_{k} m_{2 j_{k}} \sum_{i=1}^{p_{k}} b_{k, i} \sum_{j \in \Phi_{k, i}} b_{k, i, j} x_{k, i, j}^{R_{2}}\right)
$$

and

$$
\tilde{y}=\left(\sum_{k=1}^{d} b_{k} \sum_{i=1}^{p_{k}} b_{k, i} \sum_{j \in \Phi_{k, i}} b_{k, i, j} x_{k, i, j}^{R_{2}}\right)
$$

Observe that

$$
\sum_{\ell=1}^{r} \lambda_{\ell} f_{\ell}(y)=\sum_{k=1}^{d} \sum_{\ell=1}^{r} \frac{\lambda_{\ell}}{\omega\left(f_{\ell}\right)} \sum_{\beta \in R_{k}^{2} \cap \mathcal{A}_{\ell}} \prod_{\ell \prec \delta \prec \beta} \frac{\lambda_{\delta}}{\omega\left(f_{\delta}\right)} \frac{1}{\omega\left(f_{\beta}\right)} \sum_{\gamma \in S_{\beta}} \lambda_{\gamma} h_{\gamma}(y) .
$$

For each $\beta \in R_{k}^{2}$ use the fact that $\omega\left(f_{\beta}\right) \geq m_{2 j_{k}}$ to continue as follows:

$$
\sum_{\ell=1}^{r} \lambda_{\ell} f_{\ell}(y) \leq \frac{1}{\min _{1 \leq \ell \leq r} \omega\left(f_{\ell}\right)} \sum_{k=1}^{d} \frac{1}{m_{2 j_{k}}} \sum_{\ell=1}^{r} \lambda_{\ell} \sum_{\beta \in R_{k}^{2} \cap \mathcal{A}_{\ell}} \prod_{\ell \prec \delta \prec \beta} \frac{\lambda_{\delta}}{\omega\left(f_{\delta}\right)} \sum_{\gamma \in S_{\beta}} \lambda_{\gamma} h_{\gamma}(y) .
$$

Since $\bigcup_{\beta \in R_{k}^{2}} \operatorname{supp} h_{\beta}=\operatorname{supp} x_{k}^{R_{2}}$, each $m_{2 j_{k}}$ term cancels to yield

$$
\sum_{\ell=1}^{r} \lambda_{\ell} f_{\ell}(y) \leq \frac{1}{\min _{1 \leq \ell \leq r} \omega\left(f_{\ell}\right)} \sum_{\gamma \in \cup S_{\beta}} \eta_{\gamma} h_{\gamma}(\tilde{y})
$$


The final estimate follows by applying Lemma 6.2 and Sublemma 8.2 Combining the estimates (1), (2), (3), and (4) yields the result.

We now pass to show the main result of this section, namely the proof of Proposition 6.7

Proof of Proposition 6.7. For each $k \in\{1, \ldots, d\}, x_{k}$ is of the form,

$$
x_{k}=m_{2 j_{k}} \sum_{i=1}^{p_{k}} b_{k, i} x_{k, i}
$$

Note that $m_{2 j_{k}} b_{k, i}<1 / m_{2 j_{k}}$, hence $\left\|x_{k}\right\|_{\infty}<1$. We shall decompose $\{1, \ldots, r\}$ as follows:

$$
E_{0}=\left\{\ell: w\left(f_{\ell}\right)<m_{2 j_{1}}\right\}
$$

For $k \in\{1, \ldots, d\}$,

$$
E_{k, 0}=\left\{\ell: m_{2 j_{k}} \leq w\left(f_{\ell}\right)<m_{2 j_{k, 1}}\right\} .
$$

For $k \in\{1, \ldots, d\}$ and $1 \leq i<p_{k}$,

$$
E_{k, i}=\left\{\ell: m_{2 j_{k, i}} \leq w\left(f_{\ell}\right)<m_{2 j_{k, i+1}}\right\},
$$

and for $1 \leq k<d$,

$$
E_{k, p_{k}}=\left\{\ell: m_{2 j_{k, p_{k}}} \leq w\left(f_{\ell}\right)<m_{2 j_{k+1}}\right\},
$$

and finally,

$$
E_{d, p_{d}}=\left\{l: m_{2 j_{d, p_{d}}} \leq w\left(f_{\ell}\right)\right\} .
$$

Next, according to the set that some $\ell$ belongs to, we split the functional $f_{\ell}$ into at most four parts denoted as $f_{\ell}^{\varepsilon}$, with $\varepsilon=1,2,-1,-2$ as follows. Let $\ell \in E_{k, i}$ then, $f_{\ell}^{-2}=\left.f_{\ell}\right|_{\left[1, \operatorname{maxsupp} x_{k, i-1}\right]}$ if $1<i$ and $f_{\ell}^{-2}=\left.f_{\ell}\right|_{\left[1, \operatorname{maxsupp} x_{k-1}\right]}$ if $i=1$.

$f_{\ell}^{-1}=\left.f_{\ell}\right|_{\left[\operatorname{supp} x_{k, i}\right]}, f_{\ell}^{1}=\left.f_{\ell}\right|_{\left[\operatorname{minsupp} x_{k, i+1}, \operatorname{maxsupp} x_{k}\right]}$ if $i<p_{k}$ and $f_{\ell}^{1}=0$ if $i=p_{k}$. $f_{\ell}^{2}=\left.f_{\ell}\right|_{\left[\operatorname{minsupp} x_{k+1}, \infty\right)}$ if $k<d$ and $f_{\ell}^{2}=0$ if $k=d$.

For $\ell \in E_{k, 0}$ set $f_{\ell}^{-2}=\left.f_{\ell}\right|_{\left[1, \operatorname{maxsupp} x_{k-1}\right]}, f_{\ell}^{-1}=\left.f_{\ell}\right|_{\left[\operatorname{supp} x_{k}\right]}, f_{\ell}^{1}=0$ and $f_{\ell}^{2}=$ $\left.f_{\ell}\right|_{\left[\operatorname{minsupp} x_{k+1}, \infty\right)}$. Finally for $\ell \in E_{0}$ set $f_{\ell}^{-2}=f_{\ell}^{-1}=f_{\ell}^{1}=0$ and $f_{\ell}^{2}=f_{\ell}$.

We proceed to the estimates:

Notice that for $\varepsilon \in\{-2, \ldots, 2\}$,

$$
\begin{aligned}
\sum_{\ell=1}^{r} \lambda_{\ell} f_{\ell}^{\varepsilon}\left(\sum_{k=1}^{d} b_{k} x_{k}\right) & =\sum_{\ell \in E_{0}} \lambda_{\ell} f_{\ell}^{\varepsilon}\left(\sum_{k=1}^{d} b_{k} x_{k}\right)+\sum_{t=1}^{d} \sum_{\ell \in E_{t, 0}} \lambda_{\ell} f_{\ell}^{\varepsilon}\left(\sum_{k=1}^{d} b_{k} x_{k}\right) \\
& +\sum_{t=1}^{d} \sum_{j=1}^{p_{k}} \sum_{\ell \in E_{t, j}} \lambda_{\ell} f_{\ell}^{\varepsilon}\left(\sum_{k=1}^{d} b_{k} x_{k}\right) .
\end{aligned}
$$

(1) For $\ell \in E_{0}$ Proposition 8.1 yields $\sum_{\ell \in E_{0}} \lambda_{\ell} f_{\ell}^{2}\left(\sum_{k=1}^{d} b_{k} x_{k}\right) \leq \frac{16 C}{\min _{\ell} w\left(f_{\ell}\right)}$, while for $\varepsilon \neq 2$ the corresponding sum is equal to zero. 
(2) For the sum

$$
\sum_{t=1}^{d} \sum_{\ell \in E_{t, 0}} \lambda_{\ell} f_{\ell}^{-2}\left(\sum_{k=1}^{d} b_{k} x_{k}\right)+\sum_{t=1}^{d} \sum_{j=1}^{p_{k}} \sum_{\ell \in E_{t, j}} \lambda_{\ell} f_{\ell}^{-2}\left(\sum_{k=1}^{d} b_{k} x_{k}\right),
$$

we can directly apply the RISE (Lemma 6.4) and obtain

$$
\sum_{\ell=1}^{r} \lambda_{\ell} f_{\ell}^{-2}\left(\sum_{k=1}^{d} b_{k} x_{k}\right) \leq \frac{2}{m_{2 j_{1}}} \text {. }
$$

(3) Note that

$$
\sum_{t=1}^{d} \sum_{\ell \in E_{t, 0}} \lambda_{\ell} f_{\ell}^{-1}\left(\sum_{k=1}^{d} b_{k} x_{k}\right)=\sum_{k=1}^{d} m_{2 j_{k}} b_{k} \sum_{\ell \in E_{k, 0}} \lambda_{\ell} f_{\ell}^{-1}\left(\sum_{i=1}^{p_{k}} b_{k, i} x_{k, i}\right) .
$$

Observe that for all $\ell \in E_{k, 0}, w\left(f_{\ell}\right)>m_{2 j_{k}}$ if $f_{\ell}^{-1} \neq 0$ by our assumption. In addition, the family $\left(f_{\ell}^{-1}\right)_{\ell \in E_{k, 0}}$ is $S_{n_{q}}$-allowable and $w\left(f_{\ell}^{-1}\right)<m_{2 j_{k, 1}}$. Hence, Proposition 8.1 yields that

$$
\sum_{\ell \in E_{k, 0}} \lambda_{\ell} f_{\ell}^{-1}\left(\sum_{i=1}^{p_{k}} b_{k, i} x_{k, i}\right) \leq \frac{12 C}{\min _{\ell} w\left(f_{\ell}\right)} .
$$

Therefore, the estimate is a result of a summation for all $k$.

For the term $\sum_{t=1}^{d} \sum_{j=1}^{p_{k}} \sum_{\ell \in E_{t, j}} \lambda_{\ell} f_{\ell}^{-1}\left(\sum_{k=1}^{d} b_{k} x_{k}\right)$ we may apply the SAE (Lemma 6.3) to see that

$$
\sum_{j=1}^{p_{k}} \sum_{\ell \in E_{t, j}} \lambda_{\ell} f_{\ell}^{-1}\left(m_{2 j_{k}} \sum_{i=1}^{p_{k}} b_{k, i} x_{k, i}\right) \leq \frac{C}{m_{2 j_{k}}} .
$$

Again summing up for all $k$ yields the desired estimate.

(4) Notice that $\sum_{\ell \in E_{k, i}} \lambda_{\ell} f_{\ell}^{1}\left(x_{k}\right)=\sum_{\ell \in E_{k, i}} \lambda_{\ell} f_{\ell}^{1}\left(m_{2 j_{k}} \sum_{t=i+1}^{p_{k}} b_{k, t} x_{k, t}\right)$. Consequently, applying Lemma 6.6 we have

$$
\sum_{\ell \in E_{k, i}} \lambda_{\ell} f_{\ell}^{1}\left(x_{k}\right) \leq \frac{5 C m_{2 j_{k}}}{m_{2 j_{k, i}}} .
$$

Thus, summing up for all $i$ yields

$$
\sum_{i=1}^{p_{k}} \sum_{\ell \in E_{k, i}} \lambda_{\ell} f_{\ell}^{1}\left(x_{k}\right) \leq \sum_{i=1}^{p_{k}} \frac{5 C m_{2 j_{k}}}{m_{2 j_{k, i}}} \leq \frac{10 C}{m_{2 j_{k}}} .
$$

Hence, the summation for all $k$ gives the desired evaluation.

(5) Finally, for the sum $\sum_{t=1}^{d} \sum_{j=1}^{p_{k}} \sum_{\ell \in E_{t, j}} \lambda_{\ell} f_{\ell}^{2}\left(\sum_{k=1}^{d} b_{k} x_{k}\right)$ we can verify that the conditions of Proposition 8.1 are fulfilled and a direct application yields the result.

\section{REFERENCES}

[1] G. Androulakis and K. Beanland. A hereditarily indecomposable asymptotic $l_{2}$ Banach space. Glasg. Math. J., 48(3):503-532, 2006. MR2271380(2007h:46016)

[2] G. Androulakis, P. G. Casazza, and D. N. Kutzarova. Some more weak Hilbert spaces. Canad. Math. Bull., 43(3):257-267, 2000. MR1776054 (2002h:46012) 
[3] R. Anisca. An irregularity in the class of weak Hilbert spaces. (English summary) Illinois J. Math., 51(2):379-395, 2007. MR2342664 (2008m:46020)

[4] S. A. Argyros, K. Beanland, and Th. Raikoftsalis. A weak Hilbert space with few symmetries. Comptes Rendus Mathematique (Paris), 348:1293-1296, 2010. MR2745342

[5] S. A. Argyros, I. Deliyanni, D. N. Kutzarova, and A. Manoussakis. Modified mixed Tsirelson spaces. J. Funct. Anal., 159(1):43-109, 1998. MR.1654174 (2000j:46031)

[6] S. A. Argyros, I. Deliyanni, and A. Manoussakis. Distortion and spreading models in modified mixed Tsirelson spaces. Studia Math., 157(3):199-236, 2003. MR1980299 (2005f:46021)

[7] S. A. Argyros, J. Lopez-Abad, and S. Todorcevic. A class of Banach spaces with few nonstrictly singular operators. J. Funct. Anal., 222(2):306-384, 2005. MR2132394(2006a:46014)

[8] S. A. Argyros, S. Mercourakis, and A. Tsarpalias. Convex unconditionality and summability of weakly null sequences. Israel J. Math., 107:157-193, 1998. MR.1658551 (99m:46021)

[9] Spiros A. Argyros and Andreas Tolias. Methods in the theory of hereditarily indecomposable Banach spaces. Mem. Amer. Math. Soc., 170(806):vi+114, 2004. MR2053392(2005f:46022)

[10] P. G. Casazza and T. J. Shura. Tsirel'son's space, volume 1363 of Lecture Notes in Mathematics. Springer-Verlag, Berlin, 1989. With an appendix by J. Baker, O. Slotterbeck and R. Aron. MR981801 (90b:46030)

[11] I. Deliyanni and A. Manoussakis. Asymptotic $l_{p}$ hereditarily indecomposable Banach spaces. Illinois J. Math., 51(3):767-803, 2007. MR2379722(2008m:46025)

[12] S. J. Dilworth, V. Ferenczi, D. Kutzarova and E. Odell. On strongly asymptotic $l_{p}$ spaces and minimality J. Lond. Math. Soc. (2):409-419, 2007. MR2340235 (2008g:46016)

[13] A. Edgington. Some more weak Hilbert spaces. Studia Math., 100(1):1-11, 1991. MR 1130133 (93b:46028)

[14] V. Ferenczi and C. Rosendal Banach spaces without minimal subspaces J. Funct. Anal. 257(1):149-193, 2009. MR2523338 (2011b:46027)

[15] I. Gasparis. Strictly singular non-compact operators on hereditarily indecomposable Banach spaces. Proc. Amer. Math. Soc., 131(4):1181-1189 (electronic), 2003. MR1948110 (2003j:46009)

[16] W. T. Gowers. A solution to Banach's hyperplane problem. Bull. London Math. Soc., 26(6):523-530, 1994. MR1315601 (96a:46025)

[17] W. T. Gowers and B. Maurey, Banach spaces with small spaces of operators, Math. Ann., 307(4):543-568, 1997. MR1464131 (98g:46018)

[18] A. Grothendieck. La théorie des classes de Chern. Bull. Soc. Math. France, 86:137-154, 1958. MR.0116023 (22:6818)

[19] W. B. Johnson. A reflexive Banach space which is not sufficiently Euclidean. Studia Math., 55(2):201-205, 1976. MR0430756 (55:3761)

[20] W. B. Johnson. Banach spaces all of whose subspaces have the approximation property. In Seminar on Functional Analysis, 1979-1980 (French), pages Exp. No. 16, 11. École Polytech., Palaiseau, 1980. MR604397 (82d:46024)

[21] R. A. Komorowski and N. Tomczak-Jaegermann. Banach spaces without local unconditional structure. Israel J. Math., 89(1-3):205-226, 1995. MR1324462 (96g:46007)

[22] J. Lindenstrauss and L. Tzafriri, Classical Banach spaces I and II, Springer, 1996. MR0500056 (58:17766)

[23] A. Manoussakis, On the structure of a certain class of mixed Tsirelson spaces. Positivity, 5(3):193-238, 2001. MR.1836747 (2002g:46021)

[24] B. Maurey and H. P. Rosenthal. Normalized weakly null sequence with no unconditional subsequence. Studia Math., 61(1):77-98, 1977. MR0438091 (55:11010)

[25] V. D. Milman and G. Pisier. Banach spaces with a weak cotype 2 property. Israel J. Math., 54(2):139-158, 1986. MR852475 (88c:46022)

[26] N. J. Nielsen and N. Tomczak-Jaegermann. Banach lattices with property (H) and weak Hilbert spaces. Illinois J. Math., 36(3):345-371, 1992. MR1161972 (93i:46037)

[27] G. Pisier. Weak Hilbert spaces. Proc. London Math. Soc. (3), 56(3):547-579, 1988. MR931514 (89d:46022)

[28] G. Pisier. The volume of convex bodies and Banach space geometry, volume 94 of Cambridge Tracts in Mathematics. Cambridge University Press, Cambridge, 1989. MR 1036275 (91d:52005)

[29] Thomas Schlumprecht. An arbitrarily distortable Banach space. Israel J. Math., 76(1-2):8195, 1991. MR.1177333 (93h:46023) 
[30] A. Tcaciuc. On the existence of asymptotic- $l_{p}$ structures in Banach spaces. Canad. Math. Bull., 50(4):619-631, 2007. MR2364212 (2008k:46056)

[31] B. S. Tsirelson. It is impossible to imbed $\ell_{p}$ of $c_{0}$ into an arbitrary Banach space. Funkcional. Anal. i Priložen., 8(2):57-60, 1974. MR0350378(50:2871)

Department of Mathematics, Zografou Campus, National Technical University, Athens 157 80, Greece

E-mail address: sargyros@math.ntua.gr

Department of Mathematics and Applied Mathematics, Virginia Commonwealth University, Richmond, Virginia 23284

E-mail address: kbeanland@vcu.edu

Department of Mathematics, Zografou Campus, National Technical University, Athens 157 80, Greece

E-mail address: th_raik@hotmail.com 